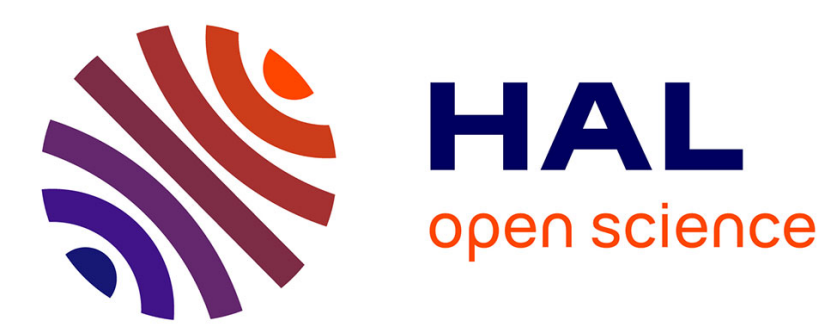

\title{
Phase-field modeling of anisotropic brittle fracture including several damage mechanisms
}

\author{
Jeremy Bleyer, Roberto Alessi
}

\section{To cite this version:}

Jeremy Bleyer, Roberto Alessi. Phase-field modeling of anisotropic brittle fracture including several damage mechanisms. Computer Methods in Applied Mechanics and Engineering, 2018, 336, pp.213236. 10.1016/j.cma.2018.03.012 . hal-01736465

\section{HAL Id: hal-01736465 \\ https://hal-enpc.archives-ouvertes.fr/hal-01736465}

Submitted on 17 Mar 2018

HAL is a multi-disciplinary open access archive for the deposit and dissemination of scientific research documents, whether they are published or not. The documents may come from teaching and research institutions in France or abroad, or from public or private research centers.
L'archive ouverte pluridisciplinaire HAL, est destinée au dépôt et à la diffusion de documents scientifiques de niveau recherche, publiés ou non, émanant des établissements d'enseignement et de recherche français ou étrangers, des laboratoires publics ou privés. 


\title{
Phase-field modeling of anisotropic brittle fracture including several damage mechanisms
}

\author{
Jeremy Bleyer ${ }^{\mathrm{a}, *}$, Roberto Alessi $^{\mathrm{b}}$ \\ ${ }^{a}$ Ecole des Ponts ParisTech, Laboratoire Navier UMR 8205 (ENPC-IFSTTAR-CNRS) \\ Université Paris-Est, Cité Descartes, 6-8 av Blaise Pascal, 77455 Champs-sur-Marne, FRANCE \\ ${ }^{b}$ Dipartimento di Ingegneria Strutturale e Geotecnica, Sapienza Università di Roma, Via Eudossiana 18, \\ 00184 Roma, ITALY
}

\begin{abstract}
The present paper aims at modeling complex fracture phenomena where different damaging mechanisms are involved. For this purpose, the standard one-variable phase-field/gradient damage model, able to regularize Griffith's isotropic brittle fracture problem, is extended to describe different degradation mechanisms through several distinct damage variables. Associating with each damage variable a different dissipated fracture energy, the coupling between all mechanisms is achieved through the degradation of the elastic stiffness. The framework is very general and can be tailored to many situations where different fracture mechanisms are present as well as to model anisotropic fracture phenomena. In this first work, after a general presentation of the model, the attention is focused on a specific paradigmatic case, namely the brittle fracture problem of a $2 \mathrm{D}$ homogeneous orthotropic medium with two different damaging mechanisms with respect to the two orthogonal directions. Illustrative numerical applications consider propagation in mode I and II as well as kinking of cracks as a result of a transition between the two fracture mechanisms. It is shown that the proposed model and numerical implementation compares well with theoretical and experimental results, allowing to reproduce specific features of crack propagation in anisotropic materials whereas standard models using one damage variable seem unable to do so.
\end{abstract}

Keywords: complex fracture, phase-field, gradient damage, anisotropic fracture, brittle fracture, variational model

${ }^{*}$ Correspondence to: J. Bleyer, Laboratoire Navier, 6-8 av Blaise Pascal, Cité Descartes, 77455 Champssur-Marne, France, Tel : +33 (0)164153704

Email addresses: jeremy.bleyer@enpc.fr (Jeremy Bleyer), roberto.alessi@uniroma1.it (Roberto Alessi)

URL: https://sites.google.com/site/bleyerjeremy/ (Jeremy Bleyer)

Preprint submitted to Computer Methods in Applied Mechanics and Engineering

March 8, 2018 


\section{Contents}

\begin{tabular}{lll}
\hline 1 & Introduction & 3
\end{tabular}

2 Phase-field approach to brittle fracture including several damage mechanisms

$2.1 \quad$ State variables and total internal material energy density . . . . . . . . . . . 4

2.2 Variational formulation . . . . . . . . . . . . . . . . . . . 5

2.3 Constitutive choices and link to brittle fracture . . . . . . . . . . . . . . . 6

$2.4 \quad$ A first example: mode mixity . . . . . . . . . . . . . . . . . . 8

3 Longitudinal/Transverse Damage (LTD) model for an orthotropic mate$\begin{array}{ll}\text { rial } & 8\end{array}$

3.1 Description of the LTD model . . . . . . . . . . . . . . . . . . . . . . . . . . . . . . . .

3.2 Damage evolution laws . . . . . . . . . . . . . . . . . . . . . . . . . 10

3.3 Comparison of LTD model with a Standard Damage (SD) model . . . . . . . 11

3.4 Advanced aspects . . . . . . . . . . . . . . . . . . . . . . . 12

$3.5 \quad$ Finite element discretization and numerical aspects . . . . . . . . . . . . . 12

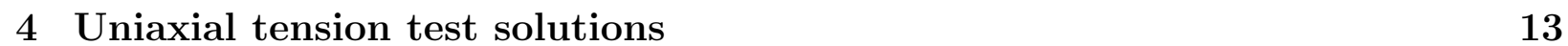

4.1 Homogeneous solutions . . . . . . . . . . . . . . . . . . . . . . . . . 13

4.2 Localized solutions . . . . . . . . . . . . . . . . . . . . . . . . . . . 14

5 Illustrative applications of the model with numerical simulations $\quad 17$

5.1 Mode I loading . . . . . . . . . . . . . . . . . . . . . . . . . . 17

5.2 Mode II loading . . . . . . . . . . . . . . . . . . . . . . . . . . . . . . . . . . . . 23

$5.3 \quad$ Crack kinking for $\alpha=90^{\circ} \ldots \ldots \ldots \ldots$. . . . . . . . . . . . . . . . . . . . 25

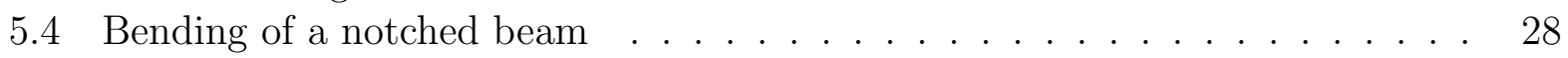

5.5 Open hole in tension $\ldots \ldots \ldots \ldots \ldots \ldots$

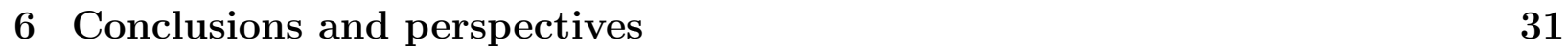

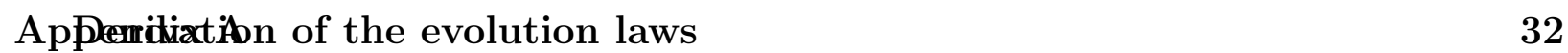




\section{Introduction}

Phase-field models of fracture have emerged as an alternative way of modeling crack propagation using a smeared representation of the crack topology. These models have been devised as a mathematical regularization of the variational approach to brittle fracture developed by Francfort and Marigo [1] which is based on the energetic competition between an elastic potential energy and the fracture dissipation, that is the work needed to create fracture surfaces, through a minimum principle. They share a conceptual resemblance with Ginzburg-Landau formulations [2, 3] of phase transitions and can also be interpreted as damage gradient models [4, 5] in which the dissipated energy density contains a regularizing damage gradient term associated with a regularizing internal length scale. The main advantage of such models is that no ad hoc crack propagation criterion needs to be introduced (contrary to XFEM approaches for instance) so that the crack evolution is based solely on energetic arguments. These approaches have been successfully applied to problems involving hydraulic fracture in porous media [6, 7], heterogeneous materials [8-11], ductile behaviors [12 15], finite deformation [16 18], thermal loading [19], fracture and debonding of thin films [20, 21], fatigue effects [22], etc. In addition to their versatility enabling their extension to many physical contexts, the predictions of such models have been validated against theoretical and experimental predictions of crack nucleation around notches [23], crack propagation in mixed-mode loading [24], cohesive fracture [25, 26], etc. They have also been shown to reproduce complex behaviors of crack propagation in a dynamic setting which have remained elusive for many other numerical methods, in particular the branching of a single crack into multiple cracks [27 29], for which a simple energetic criterion has been proposed [30], the existence of a limiting speed below the Rayleigh wave speed [30], the occurrence of a microbranching instability inherent to 3D effects [31, 32], etc.

As regards anisotropy, some works sometimes refer to the way elasticity is recovered in compression, for which different models have been developed, see for instance [29] for an extensive discussion on this subject. In order to avoid any confusion, we will refer to this aspect as tension/compression asymmetry instead of anisotropy.

An anisotropic fracture energy has already been proposed in the context of anisotropic media [33 35] and, more specifically, in the specific case of polycrystals [36, 37]. In all these models, anisotropy in the fracture energy has been introduced by enriching the quadratic gradient term with a constitutive fourth order tensor penalizing fracture interfaces in different directions, and eventually including higher-order gradient terms of the phase-field variable. Such an approach has not only the unwilling feature that the localization band width strongly depends on the crack orientation but is also mechanically arguable. In [38], an ad hoc modification of the phase-field formulation was proposed in order to simulate the anisotropy of mixed mode propagation in rocks, however, the generality of the proposed procedure does not seem obvious.

Most of these works focused essentially on the anisotropy of the fracture energy only. In the present work, we aim at tackling materials which also exhibit anisotropic elastic properties in addition to the fracture energy. More specifically, we consider a general setting 
in which the material anisotropy is due to both anisotropic elastic properties and distinct damage mechanisms, which are probably impossible to be captured with only one phasefield variable. Typical examples of such situations may involve fiber-reinforced composites, wooden materials, masonry, etc.

A literature review of existing constitutive models accounting for fracture properties of such anisotropic materials would be out of scope in this paper. Let us just mention a few works, among many others concerning fiber-reinforced composites, which proposed damage continuum mechanics models at the mesoscopic ply level [39 42]. Phase-field models have also been used to simulate the unidimensional behavior of hybrid laminates, including a competition between fracture of both layers and debonding of the adhesive interface [43].

Finally, let us mention that the following work is restricted to the case of brittle materials in which fracture is the only source of energy dissipation, excluding plasticity for instance. Nevertheless, any additional rate-independent, or even rate-dependent, phenomenon can be easily embedded in this variational setting.

Notation: Throughout this paper, the following notations will be used. Italic plain symbols correspond to scalar quantities. Vectors and second-order tensors are denoted by italic boldface symbols, e.g. $\boldsymbol{u}$ for the displacement and $\varepsilon$ for the strain. Fourth-order tensors are denoted by blackboard bold letters, e.g. $\mathbb{C}$ for the elasticity tensor and $\mathbb{I}$ for the fourth-order identity tensor. Normal boldface characters will correspond to engineering notations of second-order (resp. fourth-order) tensors in the form of vectors (resp. matrices). The inner product of second order tensors is denoted by a double dot $\varepsilon: \varepsilon=\varepsilon_{i j} \varepsilon_{j i}$. An underline symbol denotes a list, e.g. $\underline{d}=\left(d_{i}\right)_{i=1, \ldots, n}$ for a list of scalar damage variables. Time-dependence of variables will in general be omitted to ease the presentation whereas derivative with respect to the time variable will be denoted by a dot e.g. $\dot{\boldsymbol{u}}$ for the velocity.

\section{Phase-field approach to brittle fracture including several damage mechanisms}

The aim of the present paper is to model brittle crack propagation in continuum media in which fracture originates from several distinct degradation mechanisms. The proposed approach can either be constructed directly from thermodynamics assumptions using damage internal variables or, as hereafter done, as an extension of a variational gradient damage model inspired by the variational approach to brittle fracture introduced by Francfort and Marigo [1]. In this regard, we first postulate the total internal energy density of the model and specify the state variables. Then, the variational problem is set and the governing equations deduced. Finally, constitutive choices are discussed and a first example examined.

\subsection{State variables and total internal material energy density}

Let us first consider a generalization of damage gradient models used to model the behavior of quasi-brittle materials [4, 44]. In the classical formulation, the total internal material energy density, from now on called simply material energy density, is a state function of the strain $\varepsilon$, one internal scalar damage variable and its gradient. This last dependence 
induces a non-locality in the model and serves as a regularization of the ill-posed character of local damage models due to the underlying softening behavior. In the present model generalization, the material energy density $W$, still a state function, depends upon the strain, $n$ different scalar damage variables $d_{i}$ with $i=1, \ldots, n$ and their respective gradients $\nabla d_{i}$. Each damage variable is associated with a specific degradation mechanism and is assumed to vary between 0 and 1, 0 meaning that the considered damage mechanism is not activated and 1 corresponding to a fully degraded material for this specific mechanism. More precisely, we assume that the material energy density, still given by the sum of an elastic potential energy density and a state dependent dissipated energy density, can be written as follows:

$$
\begin{aligned}
W(\varepsilon, \underline{d}, \underline{\nabla d}) & =\psi(\varepsilon, \underline{d})+\delta(\underline{d}, \underline{\nabla d}) \\
& =\frac{1}{2} \varepsilon: \mathbb{C}(\underline{d}): \varepsilon+\sum_{i=1}^{n}\left(w_{i}\left(d_{i}\right)+w_{i}(1) \ell_{i}^{2} \nabla d_{i} \cdot \nabla d_{i}\right)
\end{aligned}
$$

where the elasticity tensor $\mathbb{C}$ may depend on all damage variables $\underline{d}=\left(d_{i}\right)_{i=1, \ldots, n}$. The dissipated energy density $\delta$, only due to the damaging mechanism, is written as a sum of independent contributions to each damage variable, themselves composed of a local term $w_{i}\left(d_{i}\right)$, hereafter also called damage dissipation function and a damage gradient term $w_{i}(1) \ell_{i}^{2} \nabla d_{i} \cdot \nabla d_{i}$ associated with a regularizing internal length scale $\ell_{i}$. Note that with our simplistic assumptions, the coupling between damage variables occurs only through the degradation of the elastic stiffness. However, more complex interactions between elementary damage mechanisms are possible to give rise to different constitutive behaviors.

\subsection{Variational formulation}

Let us now consider a time-dependent loading process characterized by time-dependent surface forces $\boldsymbol{F}(t)$ prescribed on the part $\partial \Omega_{T}$ of the boundary of the domain $\Omega$ and by timedependent displacements $\boldsymbol{U}(t)$ prescribed on the complementary part $\partial \Omega_{u}$ of the boundary (body forces are assumed to be zero for the sake of simplicity). At a given time $t$, the total energy of the body is given by:

$$
\mathcal{E}(\boldsymbol{u}, \underline{d} ; t)=\int_{\Omega} W\left(\nabla^{s} \boldsymbol{u}, \underline{d}, \underline{\nabla d}\right) \mathrm{d} \Omega-\int_{\partial \Omega_{T}} \boldsymbol{F} \cdot \boldsymbol{u} \mathrm{d} S
$$

with $\boldsymbol{u}=\boldsymbol{U}(t)$ on $\partial \Omega_{u}, 0 \leq d_{i}(\boldsymbol{x}) \leq 1$ in $\Omega$ and where $\nabla^{s}=\left(\nabla+\nabla^{T}\right) / 2$ denotes the symmetric part of the gradient operator.

Referring to the approach used in [45 47], evolution laws are derived from the following fundamental principles:

- damage irreversibility: We assume that at every point in $\Omega$, each damage variable is an increasing function of time:

$$
\dot{d}_{i}(\boldsymbol{x}) \geq 0 \quad \forall \boldsymbol{x} \in \Omega, \forall i=1, \ldots, n
$$


- stability condition: Let us consider the state $(\boldsymbol{u}, \underline{d})$ at time $t$ and an admissible virtual perturbation direction $(\delta \boldsymbol{u}, \delta \underline{d})$, that is, $\delta \boldsymbol{u}=0$ on $\partial \Omega_{u}$ whereas $\delta d_{i} \geq 0$ if $d_{i}<1$ and $\delta d_{i}=0$ if $d_{i}=1$. The state is said to be locally directionally stable if, for any admissible perturbation direction $(\delta \boldsymbol{u}, \delta \underline{d})$ satisfying the previous conditions, there exists $\bar{h}$ such that for all $h \in[0 ; \bar{h}]$ :

$$
\mathcal{E}(\boldsymbol{u}+h \delta \boldsymbol{u}, \underline{d}+h \delta \underline{d} ; t) \geq \mathcal{E}(\boldsymbol{u}, \underline{d} ; t)
$$

- energy balance: The energy balance principle is given as the following global condition:

$$
\frac{\mathrm{d} \mathcal{E}}{\mathrm{d} t}(\boldsymbol{u}, \underline{d})=\int_{\partial \Omega_{T}} \boldsymbol{F} \cdot \dot{\boldsymbol{u}} \mathrm{d} S+\int_{\Omega_{u}} \dot{\boldsymbol{U}} \cdot \boldsymbol{\sigma} \cdot \boldsymbol{n} \mathrm{d} S
$$

By exploiting these general principles, the following evolution laws are deduced (see Appendix A):

- equilibrium and natural boundary conditions:

$$
\begin{aligned}
\operatorname{div} \boldsymbol{\sigma}=0 \quad \text { in } \Omega \\
\boldsymbol{\sigma} \cdot \boldsymbol{n}=\boldsymbol{F} \quad \text { on } \partial \Omega_{T}
\end{aligned}
$$

- damage evolution criterion and consistency equations: $\forall i=1, \ldots, n$ and $\forall \boldsymbol{x} \in \Omega$

$$
\begin{aligned}
& Y_{i}=-\partial_{d_{i}} \psi(\boldsymbol{u}, \underline{d})-\left(w_{i}^{\prime}\left(d_{i}\right)-2 w_{i}(1) \ell_{i}^{2} \Delta d_{i}\right) \\
& Y_{i} \leq 0, \quad \dot{d}_{i} \geq 0, \quad \dot{d}_{i} Y_{i}=0
\end{aligned}
$$

Note that the boundary conditions of the damage fields on $\partial \Omega$ naturally arise from the variational formulation and are given by $\partial d_{i} / \partial n \geq 0$ and $\left(\partial d_{i} / \partial n\right) \dot{d}_{i}=0$.

The obtained evolution equations are therefore similar to the case of only one damage variable except that a coupling between all damage mechanisms is introduced by the elastic part $\partial_{d_{i}} \psi(\boldsymbol{u}, \underline{d})$. The conditions $Y_{i} \leq 0$ must therefore be simultaneously satisfied in the damage evolution problem. The issue of true stability and non-uniqueness of solutions for the evolution problem are obviously still present in this framework and we refer to [48] for more details on the matter.

\subsection{Constitutive choices and link to brittle fracture}

Many models can be considered by making specific choices for the damage-dependent elastic stiffness tensor $\mathbb{C}(\underline{d})$ and the damage dissipation function $w_{i}\left(d_{i}\right)$. The choice of $\mathbb{C}(\underline{d})$ will be discussed in section 3 in the specific setting of an orthotropic material model.

Considering the damage dissipation function, a quadratic model $w_{i}(d)=k d^{2}$ is widely used in the literature because of the original established link of phase-field models with brittle fracture [49]. However, one disadvantage of such a model is that there is no pure 
elastic phase since damage starts to evolve as soon as the material is loaded. On the contrary, among a broad class of phase-field models regularizing the brittle fracture problem [50], a linear dependence $w_{i}(d)=k d$ allows to recover an explicit elastic phase. Taking advantage of the aforementioned link, the unspecified constant $k$ can be chosen, depending on the model, as a function of the fracture energy $G_{\mathrm{c}}$, that is the energy dissipated within a fully developed damage localization, and the internal length scale $\ell$ so that localized onedimensional solutions dissipate exactly $G_{\mathrm{c}}$ [44]. For the quadratic and linear models, the damage dissipation functions are given, respectively, by:

$$
\begin{aligned}
& w_{i}(d)=\frac{G_{\mathrm{c}}^{i}}{2 \ell} d^{2} \\
& w_{i}(d)=\frac{3 G_{\mathrm{c}}^{i}}{8 \ell} d
\end{aligned}
$$

Because of the existence of a purely elastic phase, we retained the linear model (11) for all mechanisms in the subsequent simulations (see also [23] for arguments supporting such a choice). Note that, in the standard approach with one damage variable, the choice of $G_{\mathrm{c}}$ and $\ell$ induces the existence of a maximal uniaxial stress. The internal length can then either be viewed as a purely numerical regularizing parameter if one aims at modeling a nominally brittle material or as a material parameter which can be related to a critical stress for quasi-brittle materials [23]. More refined models also exist in which the critical stress can be chosen independently from $G_{\mathrm{c}}$ and $\ell$ [51].

The total dissipated energy is given by the sum of the energy dissipated by each damage mechanisms, namely

$$
\Delta(\underline{d}, \underline{\nabla d})=\int_{\Omega} \delta(\underline{d}, \underline{\nabla d}) \mathrm{d} \Omega=\sum_{i=1}^{n} G_{\mathrm{c}}^{i} \int_{\Omega} \gamma_{i}\left(d_{i}, \nabla d_{i}\right) \mathrm{d} \Omega
$$

where the damage density functions $\gamma_{i}$ are traditionally taken in the phase-field literature (up to a possible renormalization of $\ell_{i}$ ) as:

$$
\gamma_{i}\left(d_{i}, \nabla d_{i}\right)=\frac{1}{2 \ell_{i}}\left(d_{i}^{2}+\ell_{i}^{2} \nabla d_{i} \cdot \nabla d_{i}\right)
$$

for an underlying quadratic damage model according to 10$]$, or

$$
\gamma_{i}\left(d_{i}, \nabla d_{i}\right)=\frac{3}{8 \ell_{i}}\left(d_{i}+\ell_{i}^{2} \nabla d_{i} \cdot \nabla d_{i}\right)
$$

for an underlying linear damage model according to (11). For each of these choices it is possible to interpret $G_{\mathrm{c}}^{i} \gamma_{i}\left(d_{i}, \nabla d_{i}\right)$ as the fracture energy density of the corresponding mechanism and which is exactly its contribution to the total fracture energy density $\delta(\underline{d}, \underline{\nabla d})$ introduced in the damage gradient model. Under a more theoretical point of view, many questions still need to be answered. For instance, the existence of a $\Gamma$-convergence result, as the internal length scales $\ell_{i}$ go to zero, of the present proposed phase-field model towards a 
well-defined discontinuity model with sharp fracture interfaces is still an open question that would deserve to be further investigated.

For simplicity, we will now consider a unique internal length scale $\ell_{i}=\ell$ for all mechanisms but different fracture energies $G_{\mathrm{c}}^{i}$.

\subsection{A first example: mode mixity}

To conclude this section, let us give a first insight into a potential application of the previously described framework in the case of materials exhibiting different mode I and mode II fracture toughness (rocks for instance). Using different damage variables and associated fracture energies, it is possible to distinguish between both failure mechanisms. A simple way of achieving such a purpose is to split the strain energy between a spherical and deviatoric part as follows:

$$
\psi\left(\varepsilon, d_{1}, d_{2}\right)=\frac{\kappa}{2}\left(1-d_{1}\right)^{2}(\operatorname{tr} \varepsilon)^{2}+\mu\left(1-d_{2}\right)^{2} \varepsilon^{d}: \varepsilon^{d}
$$

and considering different fracture energies such that a mode I fracture energy density would be associated with the first damage variable $G_{\mathrm{I}, \mathrm{c}} \gamma\left(d_{1}, \nabla d_{1}\right)$ and a mode II fracture energy density with the second variable $G_{\mathrm{II}, \mathrm{c}} \gamma\left(d_{2}, \nabla d_{2}\right)$. Obviously this is not the only possible choice and more complex models could be easily derived from this simple proposition.

\section{Longitudinal/Transverse Damage (LTD) model for an orthotropic material}

The previous framework is now applied to the specific case of a homogeneous orthotropic medium. Such a material model can be considered, at the mesoscopic scale, as representative of a unidirectional fiber-reinforced composite ply or of a wooden material for instance. We wish to remark that our aim, in the present work, is not to accurately represent all the complex constitutive behaviors of such materials but rather to capture, with a simple model, specific features of crack propagation in anisotropic materials in terms of elastic and/or fracture properties.

\subsection{Description of the LTD model}

As mentioned, we consider a brittle orthotropic material in plane stress conditions with the principal direction, that is the strongest and stiffest direction, oriented along $\boldsymbol{e}_{1}$, making an angle $\alpha$ with respect to the global frame direction $\boldsymbol{e}_{x}$, and the secondary or transverse direction perpendicular to the principal direction, oriented along $\boldsymbol{e}_{2}$. The undamaged elastic behavior is considered as orthotropic in the material frame $\left(\boldsymbol{e}_{1}, \boldsymbol{e}_{2}\right)$. The model is built on the distinction between two different damage mechanisms:

- The first one, Figure 1a, corresponds to failure when the material is loaded in tension along the principal direction. This damage mechanism will be termed as longitudinal damage and represented by the variable $d_{1}$ and the associated fracture energy $G_{\mathrm{c}}^{1}$. In case of a unidirectional fiber-reinforced composite ply, $G_{\mathrm{c}}^{1}$ can be considered as accounting for both fiber and matrix fracture energy contributions. 
- The second mechanism, Figure 1b, corresponds to failure when the material is loaded in tension along the transverse direction. This damage mechanism will be termed as transverse damage and represented by the variable $d_{2}$ and the associated fracture energy $G_{\mathrm{c}}^{2}$. In case of a unidirectional fiber-reinforced composite ply, $G_{\mathrm{c}}^{2}$ can be considered as accounting only for the matrix fracture energy contribution.

We will further assume that damage in shear is driven by both variables although it may be possible to include a third damage mechanism specifically for shear damage (see section 2.4).
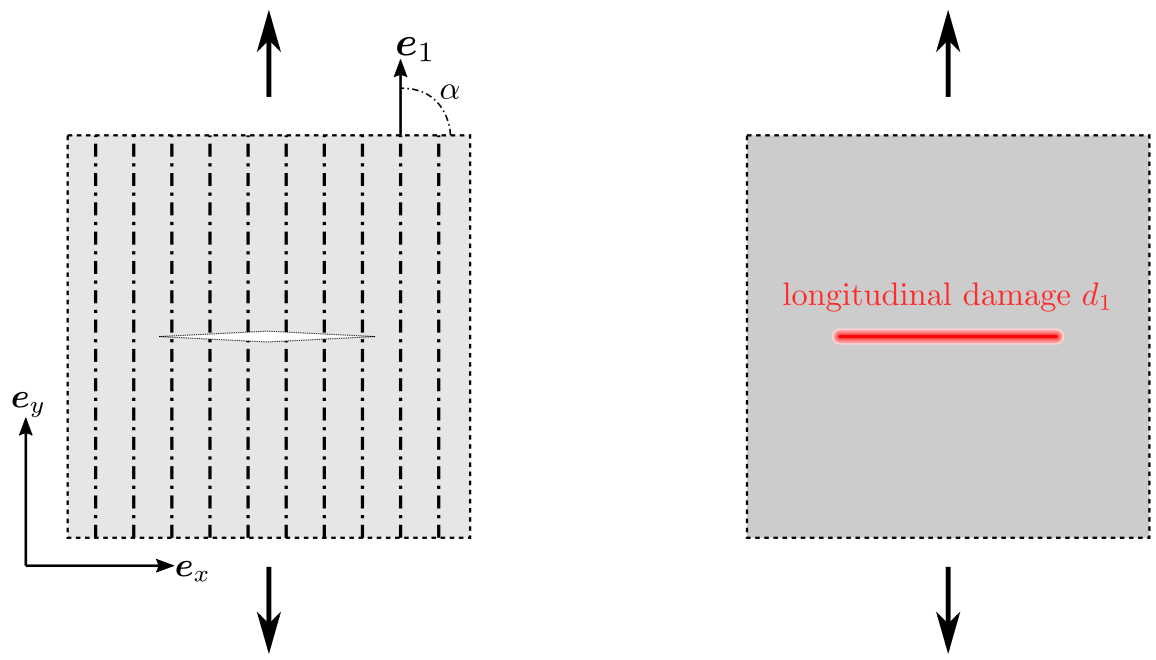

(a)
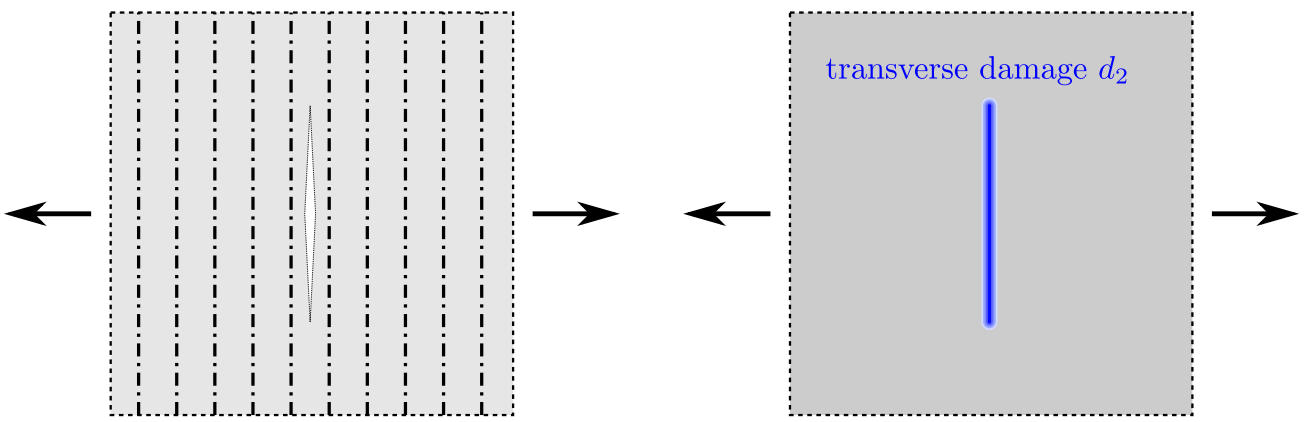

(b)

Figure 1: Longitudinal (a) and transverse (b) damage mechanisms of the LTD model. Left: sharp cracks in an orthotropic material (dashed lines indicate material orthotropy direction), right: damage localizations.

More specifically, we assume that the damage-dependent elasticity tensor is given by:

$$
\mathbb{C}(\underline{d})=\mathbb{D}(\underline{d}): \mathbb{C}^{0}: \mathbb{D}(\underline{d})
$$

where $\mathbb{C}^{0}$ is the elasticity tensor of the sound material and $\mathbb{D}(\underline{d})$ is a symmetric fourthorder damage tensor. With respect to the frame $\left(\boldsymbol{e}_{1}, \boldsymbol{e}_{2}\right)$ and taking advantage of the Voigt 
notation, the elasticity tensor $\mathbb{C}^{0}$ and the damage tensor $\mathbb{D}(\underline{d})$ are respectively represented by:

$$
\begin{aligned}
\mathbf{C}^{0} & =\left[\begin{array}{ccc}
C_{11} & C_{12} & 0 \\
C_{12} & C_{22} & 0 \\
0 & 0 & C_{66}
\end{array}\right] \\
\mathbf{D}(\underline{d}) & =\left[\begin{array}{ccc}
1-d_{1} & 0 & 0 \\
0 & 1-d_{2} & 0 \\
0 & 0 & \sqrt{\left(1-d_{1}\right)\left(1-d_{2}\right)}
\end{array}\right]
\end{aligned}
$$

so that the symmetric damage-dependent elasticity tensor $\mathbb{C}(\underline{d})$ is given by:

$$
\begin{aligned}
\mathbf{C}(\underline{d}) & =\mathbf{D}(\underline{d}) \mathbf{C}^{0} \mathbf{D}(\underline{d}) \\
& =\left[\begin{array}{ccc}
\left(1-d_{1}\right)^{2} C_{11} & \left(1-d_{1}\right)\left(1-d_{2}\right) C_{12} & 0 \\
\left(1-d_{1}\right)\left(1-d_{2}\right) C_{12} & \left(1-d_{2}\right)^{2} C_{22} & 0 \\
0 & 0 & \left(1-d_{1}\right)\left(1-d_{2}\right) C_{66}
\end{array}\right]
\end{aligned}
$$

It is expected that if a crack appears aligned along the secondary direction, it will be represented by the longitudinal damage variable $d_{1}=1$ so that $\sigma_{11}=\sigma_{12}=0$ on the crack surface (Figure 1a). On the contrary, if a crack appears along the principal direction, it is expected to be represented by the transverse damage $d_{2}=1$ so that $\sigma_{22}=\sigma_{12}=0$ (Figure 1b). In both cases, the fully degraded material will still be able to sustain uniaxial stress along the crack direction contrary to an isotropic damage model. If $d_{1}=d_{2}=d$, we obtain $\mathbb{C}(d)=(1-d)^{2} \mathbb{C}^{0}$, recovering a standard isotropic damage model and the standard phase-field formulation when $\mathbb{C}^{0}$ is isotropid ${ }^{1}$.

The contribution of both mechanisms to the total fracture energy reads:

$$
\delta(\underline{d}, \underline{\nabla d})=G_{\mathrm{c}}^{1} \gamma\left(d_{1}, \nabla d_{1}\right)+G_{\mathrm{c}}^{2} \gamma\left(d_{2}, \nabla d_{2}\right)
$$

with $\gamma(d, \nabla d)$ being given by the linear damage model (14). The Longitudinal/Transverse Damage model characterized by (16) and (18) will be further referenced as the LTD model.

\subsection{Damage evolution laws}

The total energy density for the LTD model is hence given by:

$$
W\left(\varepsilon, d_{1}, d_{2}\right)=\frac{1}{2} \epsilon^{\mathrm{T}} \mathbf{C}\left(d_{1}, d_{2}\right) \boldsymbol{\epsilon}+\frac{3 G_{\mathrm{c}}^{1}}{8 \ell}\left(d_{1}+\ell^{2} \nabla d_{1} \cdot \nabla d_{1}\right)+\frac{3 G_{\mathrm{c}}^{2}}{8 \ell}\left(d_{2}+\ell^{2} \nabla d_{2} \cdot \nabla d_{2}\right)
$$

\footnotetext{
${ }^{1}$ ignoring, at this stage, considerations regarding tension/compression asymmetry.
} 
with $\mathbf{C}\left(d_{1}, d_{2}\right)$ given by 17$)$ and with the vector of strain components $\boldsymbol{\epsilon}=\left\{\begin{array}{lll}\varepsilon_{11} & \varepsilon_{22} & 2 \varepsilon_{12}\end{array}\right\}^{\mathrm{T}}$ in the material frame. Introducing the following partial derivatives:

$$
\begin{aligned}
\mathbf{C}_{, 1}\left(d_{1}, d_{2}\right)=-\frac{\partial \mathbf{C}\left(d_{1}, d_{2}\right)}{\partial d_{1}}= & {\left[\begin{array}{ccc}
2\left(1-d_{1}\right) C_{11} & \left(1-d_{2}\right) C_{12} & 0 \\
\left(1-d_{2}\right) C_{12} & 0 & 0 \\
0 & 0 & \left(1-d_{2}\right) C_{66}
\end{array}\right] } \\
\mathbf{C}_{, 2}\left(d_{1}, d_{2}\right)=-\frac{\partial \mathbf{C}\left(d_{1}, d_{2}\right)}{\partial d_{2}}= & {\left[\begin{array}{ccc}
0 & \left(1-d_{1}\right) C_{12} & 0 \\
\left(1-d_{1}\right) C_{12} & 2\left(1-d_{2}\right) C_{22} & 0 \\
0 & 0 & \left(1-d_{1}\right) C_{66}
\end{array}\right] }
\end{aligned}
$$

the damage evolution laws (8)-(9) of the LTD model are given by:

$$
\begin{aligned}
& \left\{\begin{array}{l}
Y_{1} \leq 0 \\
\dot{d}_{1} \geq 0 \\
\dot{d}_{1} Y_{1}=0
\end{array} \quad \text { with } \quad Y_{1}=-\partial_{d_{1}} W=\frac{1}{2} \boldsymbol{\epsilon}^{\mathrm{T}} \mathbf{C}_{, 1}\left(d_{1}, d_{2}\right) \boldsymbol{\epsilon}-\frac{3 G_{\mathrm{c}}^{1}}{8 \ell}\left(1-2 \ell^{2} \Delta d_{1}\right)\right. \\
& \left\{\begin{array}{l}
Y_{2} \leq 0 \\
\dot{d}_{2} \geq 0 \\
\dot{d}_{2} Y_{2}=0
\end{array} \quad \text { with } \quad Y_{2}=-\partial_{d_{2}} W=\frac{1}{2} \boldsymbol{\epsilon}^{\mathrm{T}} \mathbf{C}_{, 2}\left(d_{1}, d_{2}\right) \boldsymbol{\epsilon}-\frac{3 G_{\mathrm{c}}^{2}}{8 \ell}\left(1-2 \ell^{2} \Delta d_{2}\right)\right.
\end{aligned}
$$

\subsection{Comparison of LTD model with a Standard Damage (SD) model}

In the following, the previous LTD model will be compared to a Standard Damage model (SD). More specifically the SD model considers only one scalar damage variable $d$ with $\mathbf{C}(d)=(1-d)^{2} \mathbf{C}^{0}$ (hence, it corresponds to the LTD model for which $d_{1}=d_{2}=d$ in (17) ) and a total fracture energy $G_{\mathrm{c}}$ associated with a fracture energy density given by:

$$
\delta(d, \nabla d)=G_{\mathrm{c}} \gamma(d, \nabla d)
$$

Note that this single-parameter damage model is isotropic with respect to the fracture energy whereas it is still anisotropic due to the elastic stiffness anisotropy. Besides, even in the case when equal fracture toughnesses are considered for the LTD model, namely $G_{\mathrm{c}}^{1}=G_{\mathrm{c}}^{2}=G_{\mathrm{c}}$, both models are expected to behave differently due to the possible independent evolution of $d_{1}$ and $d_{2}$ in the LTD model (22)-(23). Such differences will be further illustrated in section 5. The evolution damage law for this model then reads:

$$
\left\{\begin{array}{l}
Y \leq 0 \\
\dot{d} \geq 0 \\
\dot{d} Y=0
\end{array} \quad \text { with } \quad Y=\frac{1}{2}(1-d) \boldsymbol{\epsilon}^{\mathrm{T}} \mathbf{C}^{0} \boldsymbol{\epsilon}-\frac{3 G_{\mathrm{c}}}{8 \ell}\left(1-2 \ell^{2} \Delta d\right)\right.
$$

The SD model can also be modified to consider an anisotropic fracture energy (with $G_{c}^{1} \neq G_{c}^{2}$ ) by including an anisotropic gradient term as follows:

$$
\delta(d, \nabla d)=\frac{3 G_{\mathrm{c}}^{1}}{8 \ell}\left(d+\ell^{2} \nabla d \cdot \boldsymbol{B} \cdot \nabla d\right)
$$


where $\boldsymbol{B}=\boldsymbol{e}_{1} \otimes \boldsymbol{e}_{1}+\chi \boldsymbol{e}_{2} \otimes \boldsymbol{e}_{2}$ is an anisotropic tensor and $\chi=G_{\mathrm{c}}^{2} / G_{\mathrm{c}}^{1}$. This type of fracture energy anisotropy has already been considered in other works [35, 37]. It ensures that for a crack propagating perpendicularly to the principal direction, i.e. $\nabla d$ is along $\boldsymbol{e}_{1}$, the fracture energy is $G_{\mathrm{c}}^{1}$, whereas for a crack propagating along the principal direction, i.e. $\nabla d$ is along $\boldsymbol{e}_{2}$, the fracture energy is $\chi G_{\mathrm{c}}^{1}=G_{\mathrm{c}}^{2}$. One drawback of such an approach is that, for cracks parallel to the principal direction, the regularized length is $\ell$, whereas, along the secondary direction, it becomes $\sqrt{\chi} \ell$. In addition, it is still not clear what such a gradient energy contribution does physically represent.

\subsection{Advanced aspects}

Similarly to standard phase-field approaches, the elastic energy density (16) will be modified by first introducing a small residual stiffness $k_{\text {res }}$ for quasi-static simulations in order to avoid non-invertible stiffness when reaching a fully damaged state. To this end, the tensor $\mathbb{D}(\underline{d})$ in $(16)$ is replaced by $\widetilde{\mathbb{D}}(\underline{d})=\left(1-k_{\text {res }}\right) \mathbb{D}(\underline{d})+k_{\text {res }} \mathbb{I}$ where $k_{\text {res }}$ is typically $\approx 10^{-6}$.

Secondly, tension/compression asymmetry can also be introduced to avoid damage evolution in compression. If different ways of distinguishing between tension and compression exist for isotropic models [29, 52], the situation is here clearer due to the preferential direction induced by the material orthotropic directions. The elastic energy density can therefore be split depending on the positive/negative part of the longitudinal $\varepsilon_{11}$ or transverse strain $\varepsilon_{22}$ as follows:

$$
\psi(\boldsymbol{\varepsilon}, \underline{d})=\frac{1}{2}\left(\boldsymbol{\epsilon}^{+}\right)^{\mathrm{T}} \widetilde{\mathbf{D}}(\underline{d}) \mathbf{C}^{0} \widetilde{\mathbf{D}}(\underline{d}) \boldsymbol{\epsilon}^{+}+\frac{1}{2}\left(\boldsymbol{\epsilon}^{-}\right)^{\mathrm{T}} \mathbf{C}^{0} \boldsymbol{\epsilon}^{-}
$$

where

$$
\boldsymbol{\epsilon}^{+}=\left\{\begin{array}{c}
\left\langle\varepsilon_{11}\right\rangle_{+} \\
\left\langle\varepsilon_{22}\right\rangle_{+} \\
2 \varepsilon_{12}
\end{array}\right\}, \quad \boldsymbol{\epsilon}^{-}=\left\{\begin{array}{c}
\left\langle\varepsilon_{11}\right\rangle_{-} \\
\left\langle\varepsilon_{22}\right\rangle_{-} \\
0
\end{array}\right\}
$$

and $\langle\star\rangle_{ \pm}=(\star \pm|\star|) / 2$ denotes the positive/negative part of $\star$. Splitted strain projection operators can also be easily adapted from [10] to avoid the non-linearity of the previous decomposition.

\subsection{Finite element discretization and numerical aspects}

The finite element discretization relies on the FEniCS finite element library [53, 54], linear interpolation has been chosen for the displacement and damage fields. The damage evolution problem (9) is solved using the TAO bound-constrained optimization solver [55] integrated into the PETSc library [56]. The solution strategy follows an incremental energy minimization scheme [49, 57, 58]. More specifically, as regards the resolution of a given load increment, the alternate minimization algorithm is adopted and a convergence criterion given by $\left(\mathcal{E}^{k+1}-\mathcal{E}^{k}\right) / \mathcal{E}^{k}<$ tol where $\mathcal{E}^{k}$ is the total energy computed at iteration $k$ is used. In particular, no path-following scheme is used so that snap-back of unstable solutions is not captured. Mesh sizes have been taken sufficiently smaller than $\ell$ to resolve the regularization length scale (typically 4 to 5 elements for $\ell$ ). The numerical code used in this work is available at https://doi .org/10.5281/zenodo.1188970 [59]. 


\begin{tabular}{cccccc}
\hline & \multicolumn{3}{c}{ Elastic properties } & & \multicolumn{2}{c}{ Fracture properties } \\
\hline$E_{1}$ & $E_{2}$ & $\nu_{12}$ & $\mu_{12}$ & $G_{\mathrm{c}}^{1} / \ell$ & $G_{\mathrm{c}}^{2} / \ell$ \\
\hline 15 or $150 \mathrm{GPa}$ & $10 \mathrm{GPa}$ & 0.25 & $5 \mathrm{GPa}$ & $1 \mathrm{kPa}$ & $1 \mathrm{kPa}$ \\
\hline
\end{tabular}

Table 1: Material properties of the phase-field model

\section{Uniaxial tension test solutions}

\subsection{Homogeneous solutions}

In this subsection, homogeneous solutions are investigated in the case of a uniaxial tensile stress state oriented along direction $\boldsymbol{e}_{x}$. For illustrative purposes, material parameters are given in Table 1 and $G_{\mathrm{c}}=G_{\mathrm{c}}^{1}=G_{\mathrm{c}}^{2}$.

Considering an initially undamaged material and a uniaxial stress state $\boldsymbol{\sigma}=\sigma \boldsymbol{e}_{x} \otimes \boldsymbol{e}_{x}$ expressed in the material reference frame by $\boldsymbol{\sigma}=\sigma \mathbf{q}_{\alpha}$ with $\mathbf{q}_{\alpha}=\sigma\left\{\cos ^{2} \alpha \sin ^{2} \alpha-\cos \alpha \sin \alpha\right\}^{\mathrm{T}}$, the evolution criterion of the SD model (25), assuming a homogeneous solution, reduces to:

$$
Y=S(\alpha) \sigma^{2}-3 G_{\mathrm{c}} /(8 \ell) \leq 0 \Rightarrow \sigma \leq \sigma_{\mathrm{c}}=\sqrt{\frac{3 G_{\mathrm{c}}}{8 \ell S(\alpha)}}
$$

where $S(\alpha)=\mathbf{q}_{\alpha}^{\mathrm{T}} \mathbf{S}^{0} \mathbf{q}_{\alpha}$ is the uniaxial compliance of the sound material in direction $\boldsymbol{e}_{x}$ and with $\mathbf{S}^{0}=\left(\mathbf{C}^{0}\right)^{-1}$ being the full undamaged compliance tensor.

Similarly, for the LTD model, (22)-(23) reduce to:

$$
\begin{aligned}
& Y_{1}=\frac{1}{2} \boldsymbol{\epsilon}^{\mathrm{T}} \mathbf{C}_{, 1}(0,0) \boldsymbol{\epsilon}-\frac{3 G_{\mathrm{c}}^{1}}{8 \ell} \leq 0 \\
& Y_{2}=\frac{1}{2} \boldsymbol{\epsilon}^{\mathrm{T}} \mathbf{C}_{, 2}(0,0) \boldsymbol{\epsilon}-\frac{3 G_{\mathrm{c}}^{2}}{8 \ell} \leq 0
\end{aligned}
$$

Again, with $\boldsymbol{\sigma}=\sigma \mathbf{q}_{\alpha}=\mathbf{C}^{0} \boldsymbol{\epsilon}$, we have:

$$
\left\{\begin{array}{l}
S_{1}(\alpha) \sigma^{2} \leq 3 G_{\mathrm{c}}^{1} /(8 \ell) \\
S_{2}(\alpha) \sigma^{2} \leq 3 G_{\mathrm{c}}^{2} /(8 \ell)
\end{array} \Rightarrow \sigma \leq \sigma_{\mathrm{c}}=\min \left\{\sqrt{\frac{3 G_{\mathrm{c}}^{1}}{8 \ell S_{1}(\alpha)}} ; \sqrt{\frac{3 G_{\mathrm{c}}^{2}}{8 \ell S_{2}(\alpha)}}\right\}\right.
$$

with the partial uniaxial compliances $S_{i}(\alpha)=\frac{1}{2} \mathbf{q}_{\alpha}^{\mathrm{T}} \mathbf{S}^{0} \mathbf{C}_{, i}(0,0) \mathbf{S}^{0} \mathbf{q}_{\alpha}$ of the sound material.

The critical stresses given by the LTD model are higher than those of the SD model (Figure 2a) due to the independent activation of either the principal criterion $Y_{1}=0$ (in blue) or the secondary criterion $Y_{2}=0$ (in orange). The same behavior for a large orthotropy ratio $\left(E_{1} / E_{2}=15\right)$, typical of fiber-reinforced composites, is observed in Figure 2b, however 
the transition angle between activation of the principal criterion and the secondary criterion is smaller (around $27^{\circ}$ in this case compared to approximately $42^{\circ}$ for a smaller orthotropy ratio). Figure 3 represents the evolution of $2 \mathrm{D}$ elastic domains for biaxial stress states (zero shear $\left.\sigma_{x y}=0\right)$ in the case of the principal direction oriented at $\alpha=45^{\circ}$ for $E_{1} / E_{2}=15$. Depending on the evolution of each damage variable, the elastic domain changes its size and shape in a rather complex manner.

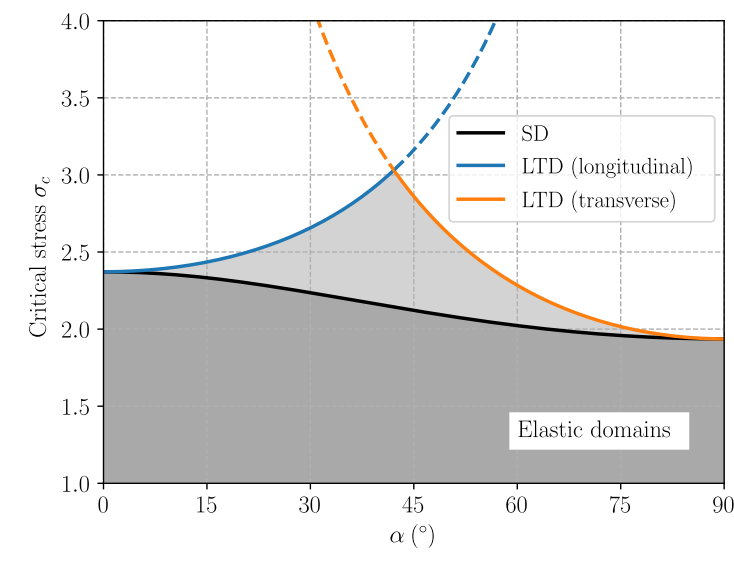

(a) $E_{1}=15 \mathrm{GPa}$

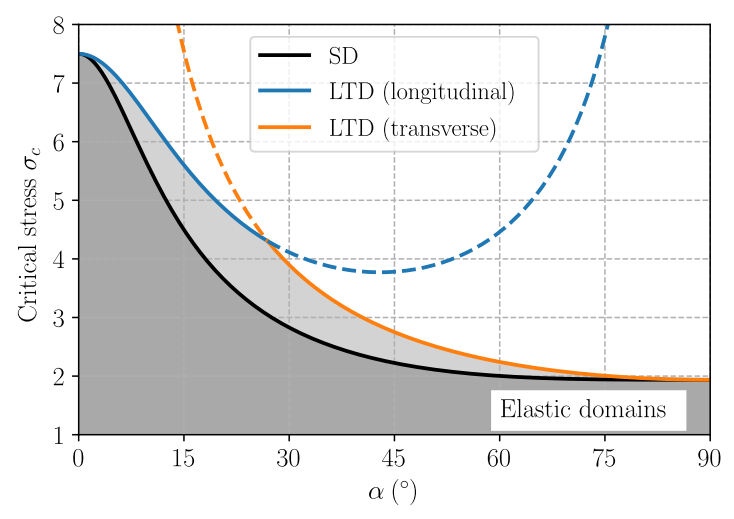

(b) $E_{1}=150 \mathrm{GPa}$

Figure 2: Uniaxial elastic domain as a function of principal orthotropic direction orientation $\alpha$ for both LTD and SD models depending on the level of orthotropy

\subsection{Localized solutions}

In this subsection, the occurrence of localized solutions is investigated for the case of a thin elongated bar under uniform tension. Standard phase-field models for isotropic media are known to exhibit a solution in which damage localizes on a narrow band orthogonal to the loading direction when the bar is sufficiently long [4]. Because we aim at investigating the influence of a principal direction misalignment with respect to the loading direction, the bar in tension has been modeled as a 2D domain of length $L=1 \mathrm{~m}$ and width $W=0.02 \mathrm{~m}$ for a regularization length of $\ell=2 \mathrm{~mm}$. The bar has been taken as sufficiently long so that homogeneous solutions are not stable and a slightly smaller section is introduced in the middle of the sample to induce damage localization around this point. Elastic properties are those of section 4.1 whereas fracture energy is taken for now as isotropic of value $G_{\mathrm{c}}^{1}=G_{\mathrm{c}}^{2}=200 \mathrm{~N} / \mathrm{m}$.

The resolution of this problem using the LTD model for varying orientations $\alpha$ with respect to the horizontal tensile direction yielded a localized band with varying orientation (Figure 4a). More precisely, for orientations close to the horizontal direction, the longitudinal damage variable $d_{1}$ is activated ( $d_{2}$ remaining to zero) and the localized band inclination $\theta$ follows the secondary direction $\alpha+90^{\circ}$. Conversely, for larger values of $\alpha$, the transverse damage variable $d_{2}$ is activated and the localized band aligns with the principal direction. 


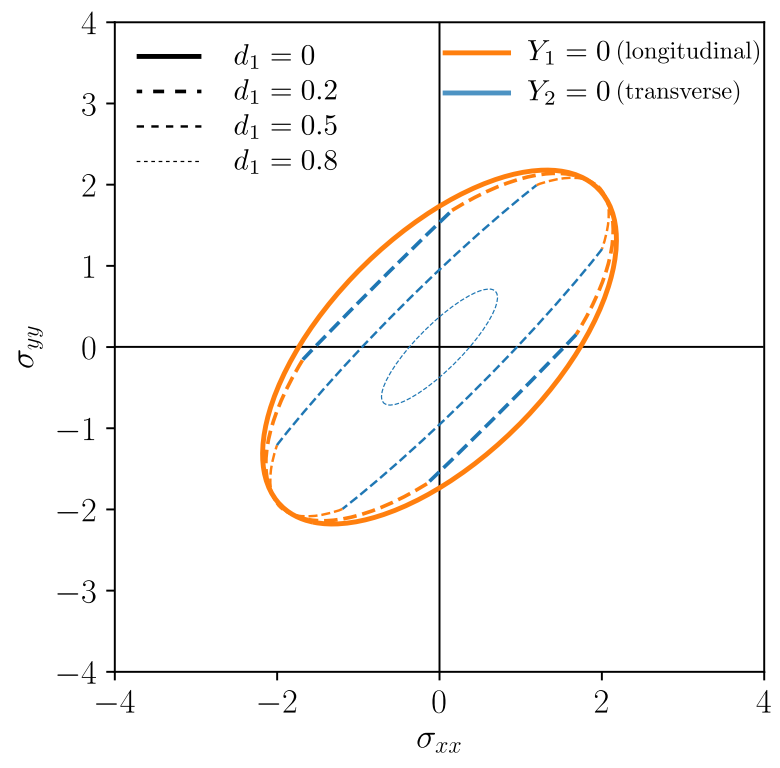

(a) Evolution for increasing longitudinal damage $d_{1}$ and $d_{2}=0$

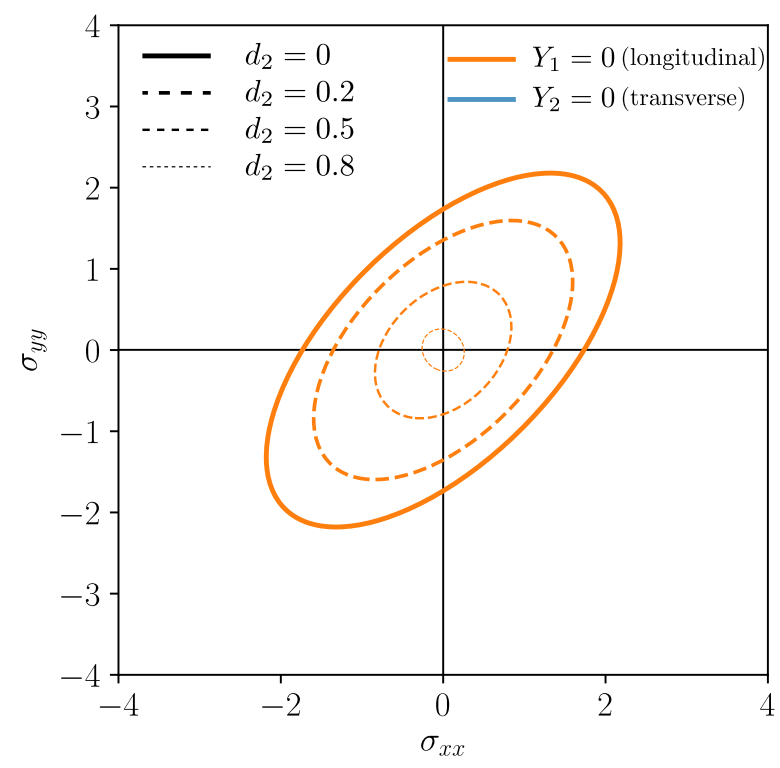

(b) Evolution for increasing transverse damage $d_{2}$ and $d_{1}=0$

Figure 3: Elastic domains for biaxial loadings $\left(\sigma_{x y}=0\right)$ and for different increasing damaging states $d_{1}$ or $d_{2}$ with $\alpha=45^{\circ}$ as fibers orientation.

The transition angle between both situations is in very good agreement with the transition found from the critical uniaxial stress of Figure 2 , that is around $42^{\circ}$ for $E_{1}=15 \mathrm{GPa}$ and around $27^{\circ}$ for $E_{1}=150 \mathrm{GPa}$. For the SD model, inclination of the localized band is weakly dependent on the principal orientation as it varies smoothly between $90^{\circ}$ and $110^{\circ}$. Finally, it seems that the localized band is wider for off-axis principal orientations, inducing an artificial overestimation of the dissipated energy [45]. This effect disappears for vanishing mesh sizes.

As initially designed, the LTD model hence yields, for uniaxial tensile loading, localized damage bands that align either with the principal direction or with the secondary direction depending on the inclination of the tensile direction. The transition between both regimes is dictated by the threshold obtained from the uniaxial critical stress computation (32). It is interesting to notice that localized solutions are very different between the LTD and SD models due to the very different damage evolution equations $(22)-(23)$ and $(25)$.

When considering an anisotropic fracture energy, e.g. with $G_{\mathrm{c}}^{1}$ much larger than $G_{\mathrm{c}}^{2}$, the overall behavior is the same except that the transition occurs for much smaller angles. Hence, as expected, cracks tend to localize parallel to the principal direction for small misalignment with respect to the horizontal direction, meaning that transverse cracks are energetically more favorable than longitudinal cracks. In the case of isotropic elastic properties but 


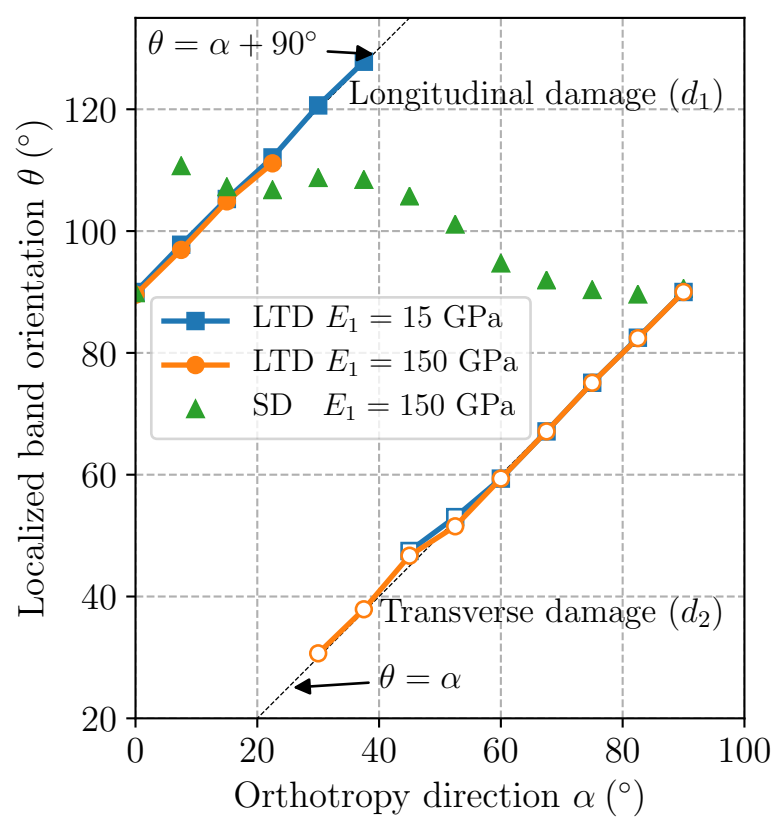

(a) Localized band orientation $\theta$

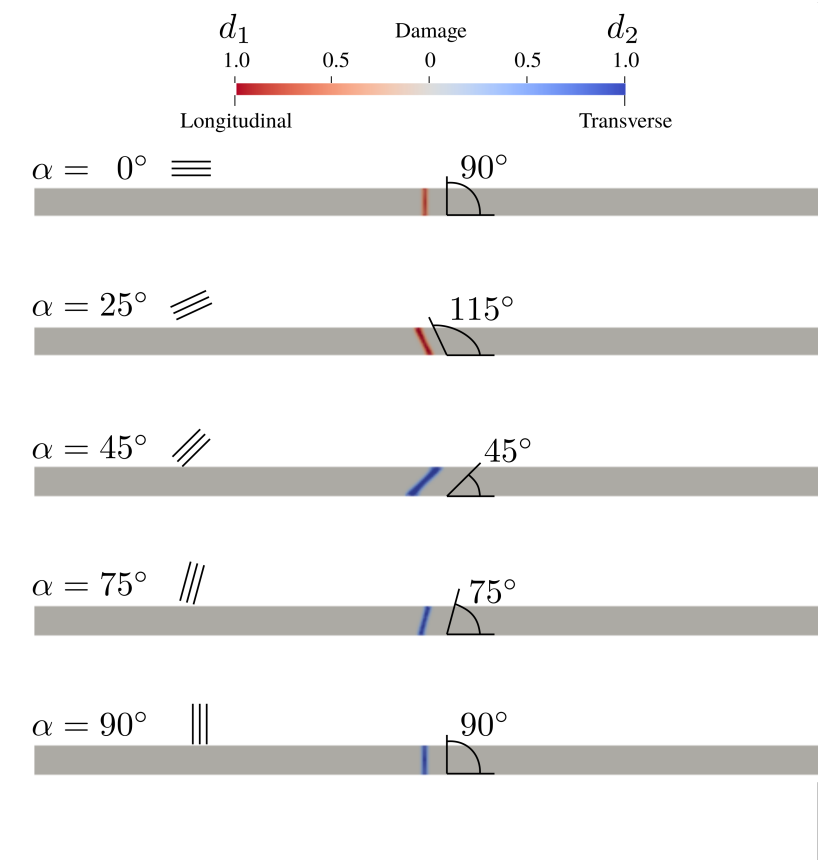

(b) Damage fields for the LTD model

Figure 4: Orientation $\theta$ of the localized band for uniaxial tension as a function of material orientation with the LTD model: a localized band for the longitudinal damage variable $d_{1}$ appears at $\theta=\alpha+90^{\circ}$ (filled symbols) for low inclination $\alpha$, localized band for the transverse damage variable $d_{2}$ appear at $\theta=\alpha$ (open symbols) for larger inclinations. Inclination of the localized band in the SD model (triangles) shows a completely different dependence on $\alpha$.

different fracture energies, a similar behavior is observed but with larger transition angles. Table 2 summarizes the observed transition angles for different ratios of fracture energies in both cases. 


\begin{tabular}{ccc}
\hline$G_{\mathrm{c}}^{1} / G_{\mathrm{c}}^{2}$ & Orthotropic elasticity & Isotropic elasticity \\
\hline 1 & $27^{\circ}$ & $47^{\circ}$ \\
5 & $13^{\circ}$ & $24^{\circ}$ \\
20 & $5^{\circ}$ & $13^{\circ}$ \\
\hline
\end{tabular}

Table 2: Approximate transition angles between longitudinal damage and transverse damage for uniaxial tension with the LTD model. Orthotropic elasticity corresponds to parameters of Table 1 with $E_{1}=150 \mathrm{GPa}$ and isotropic elasticity to $E=150 \mathrm{GPa}$ and $\nu=0.25$.

\section{Illustrative applications of the model with numerical simulations}

\subsection{Mode I loading}

We first investigate the case of a precracked domain loaded in tension by an imposed vertical displacement $U$ (Figure 5). The square plate dimension is taken as $L=1 \mathrm{~m}$ whereas the regularization length is $\ell=10 \mathrm{~mm}$, unless otherwise stated. Other material properties are those of Table 1 with $E_{1}=150 \mathrm{GPa}$.



Figure 5: Geometry of the mode I problem and boundary conditions

This problem has first been solved using the LTD model for varying principal direction orientations. In each case, an unstable crack propagation after a first elastic stage has been observed. The resulting crack paths are represented in Figure6. Except for $\alpha=90^{\circ}$, all configurations lead to a transverse crack only $\left(d_{1}=0\right.$ everywhere except close to the notch tip). It can be observed that the crack is horizontal for $\alpha=0^{\circ}$ as expected whereas for off-axis principal direction orientations the crack path follows more or less the principal direction. Crack orientation is found to be around $23^{\circ}$ for $\alpha=30^{\circ}$ and around $47^{\circ}$ for $\alpha=60^{\circ}$. For the case $\alpha=90^{\circ}$, a horizontal longitudinal crack is obtained. Contrary to what has been obtained for uniaxial tension test, the profile of the localized damage band is similar for 
all crack orientations and agrees with the analytical profile of standard phase-field models. When inspecting the failure mechanism, separation occurs in a direction normal to the crack direction (the top part is free to slide horizontally), so that failure indeed occurs in mode I. Interestingly, no situation was found in which both damage variables evolved simultaneously, a sudden transition from transverse cracking to longitudinal cracking occurred for a fibers orientation angle of approximately $78^{\circ}$, with transverse (resp. longitudinal) cracking occurring for $\alpha<78^{\circ}$ (resp. $\alpha \geq 78^{\circ}$ ), see Figure 7 .

This problem has also been solved using the SD model for which crack paths have been represented in Figure 8. Remarkably, the crack path are completely different from those predicted by the LTD model, testifying the deep differences of the damage criteria associated with each model. In particular, the dependence of the crack orientation on the principal orientation is much weaker for the SD model compared to the LTD model. It can be observed that, for all cases, unstable crack propagation occurs at a later stage for the LTD model than for the SD model. This difference between both models is especially important for a principal direction which is neither parallel nor perpendicular to the precrack. Interestingly, the LTD model predicts a larger critical displacement for $\alpha=30^{\circ}$ than $\alpha=0^{\circ}$ contrary to the SD model which predicts a decreasing critical displacement as function of $\alpha$ (Figure 9). 


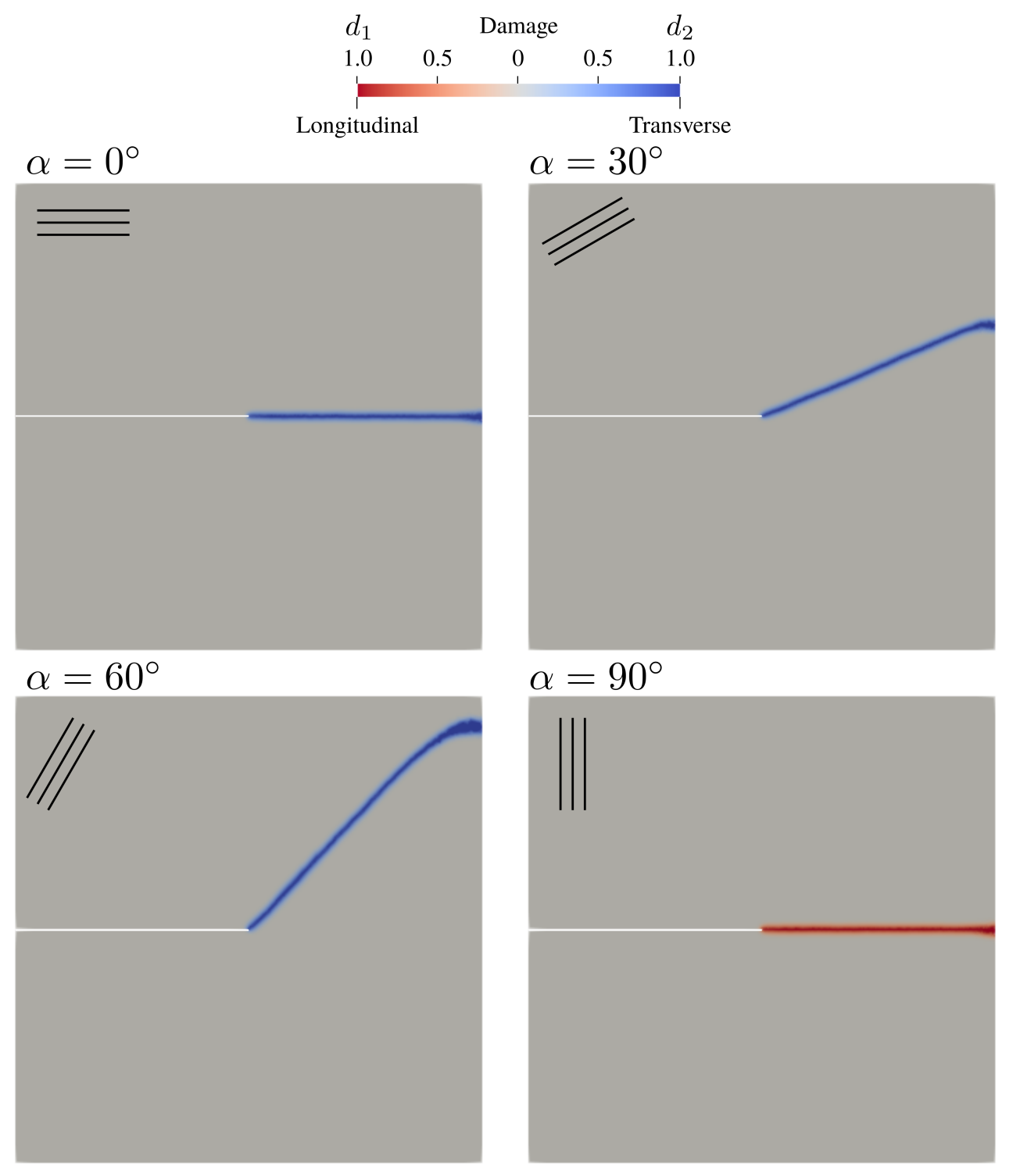

Figure 6: Crack path for mode I loading with the LTD model for different principal orthotropic direction orientations $\alpha$. Transverse cracks (damage variable $d_{2}$ ) are shown in blue, longitudinal cracks (damage variable $d_{1}$ ) are shown in red. 


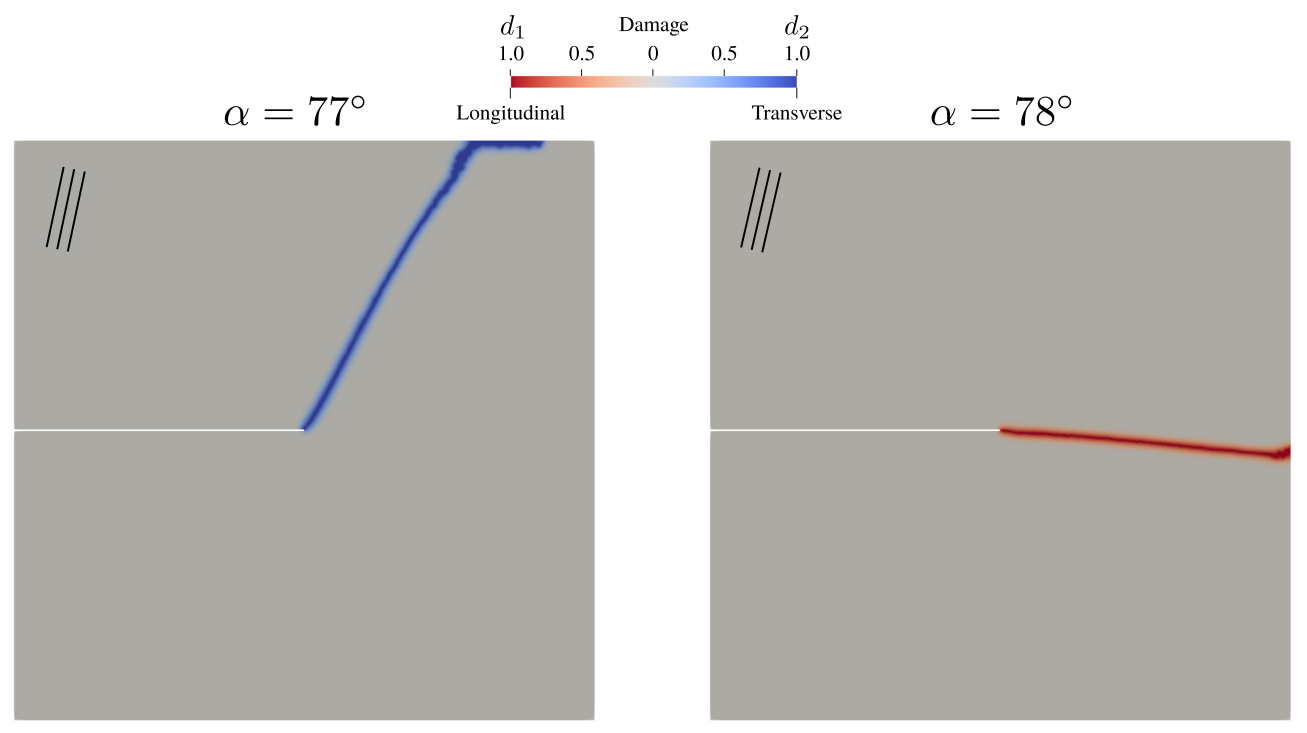

Figure 7: Transition from transverse cracking to longitudinal cracking around the critical angle 


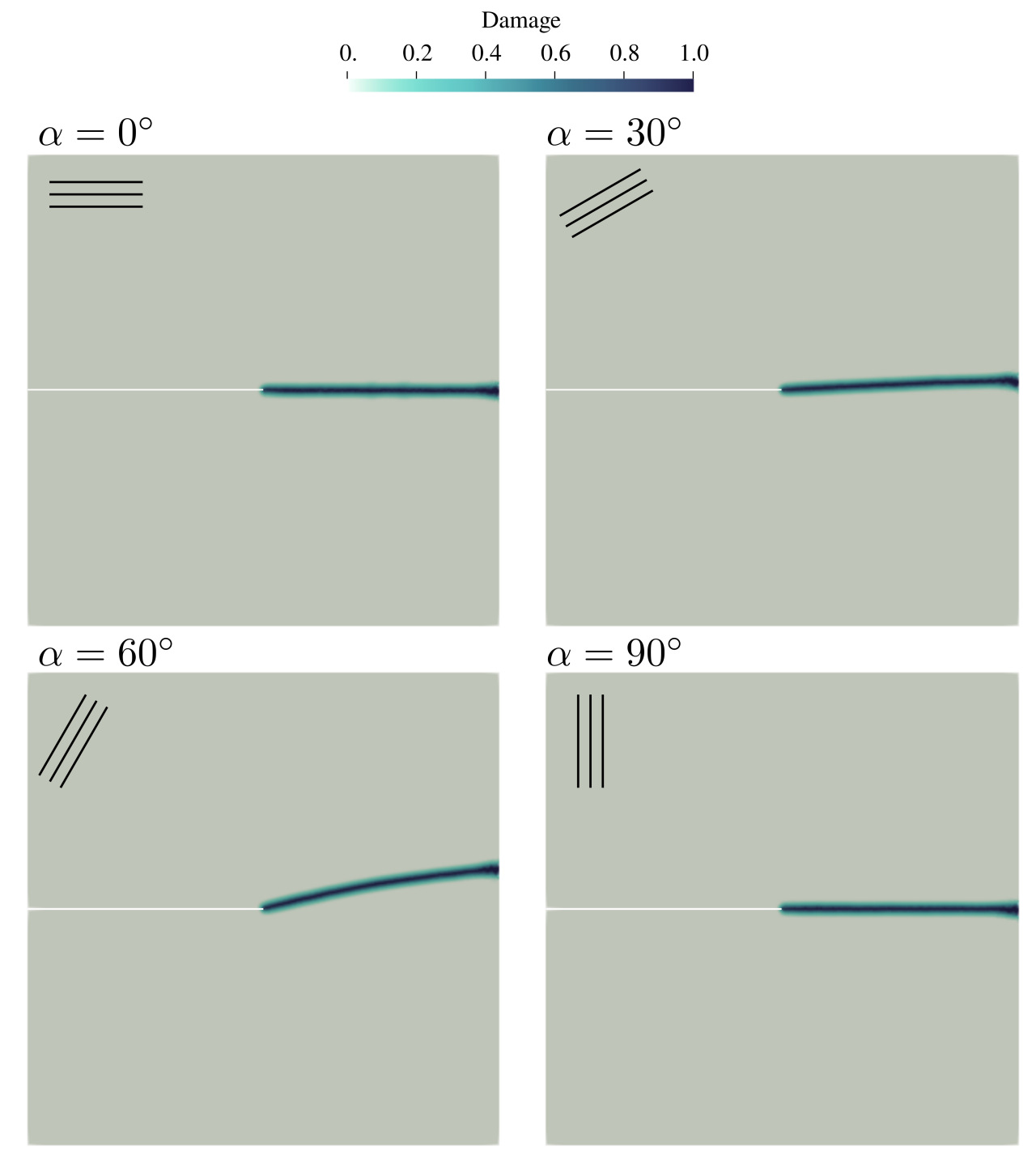

Figure 8: Crack path for mode I loading with the SD model for varying principal orthotropic direction orientation $\alpha$ 




Figure 9: Traction-displacement curves for mode I loading with varying principal orthotropic direction orientation $\alpha$. Solid lines correspond to the LTD model, dashed lines to the SD model. 


\subsection{Mode II loading}

The same problem in which a positive displacement $U$ is prescribed on the top surface along the $x$-direction $\left(\boldsymbol{u}_{y}\right.$ being free) is now considered (Figure 10). In isotropic materials, the crack is supposed to kink at an angle of approximately $-75^{\circ}$ so as to restore (more or less) a mode I loading, see [24, 60] for a discussion on different criteria predicting crack orientation and the relation with respect to standard phase field models. In anisotropic materials, crack orientation is obviously strongly influenced by the principal direction orientation, as already illustrated in the previous section. Figure 11 represents the different crack paths obtained with the LTD model for the mode II loading and different principal directions.

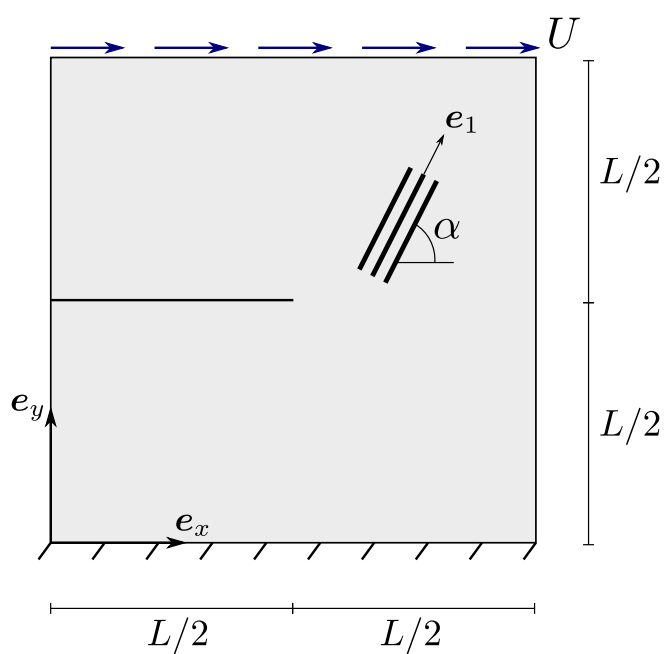

Figure 10: Geometry of the mode II problem and boundary conditions.



Figure 11: Crack path of the LTD model for the mode II loading with varying principal orthotropic direction orientation 
In particular, the case of $\alpha=0^{\circ}$ shows that the crack is able to propagate horizontally along the preferential principal direction, after a first small initial deviation. Contrary to the case of isotropic materials, a pure mode II propagation is therefore possible for such materials. Indeed, the principle of local symmetry (PLS), stating that a crack propagates in a direction such as to be in pure mode I i.e. $K_{\mathrm{II}}=0$, does not hold for orthotropic materials [60, 61]. Once again, the SD model is unable to reproduce such a behavior (see Figure 12 -center) since the crack propagates with an angle of approximately $-30^{\circ}$.

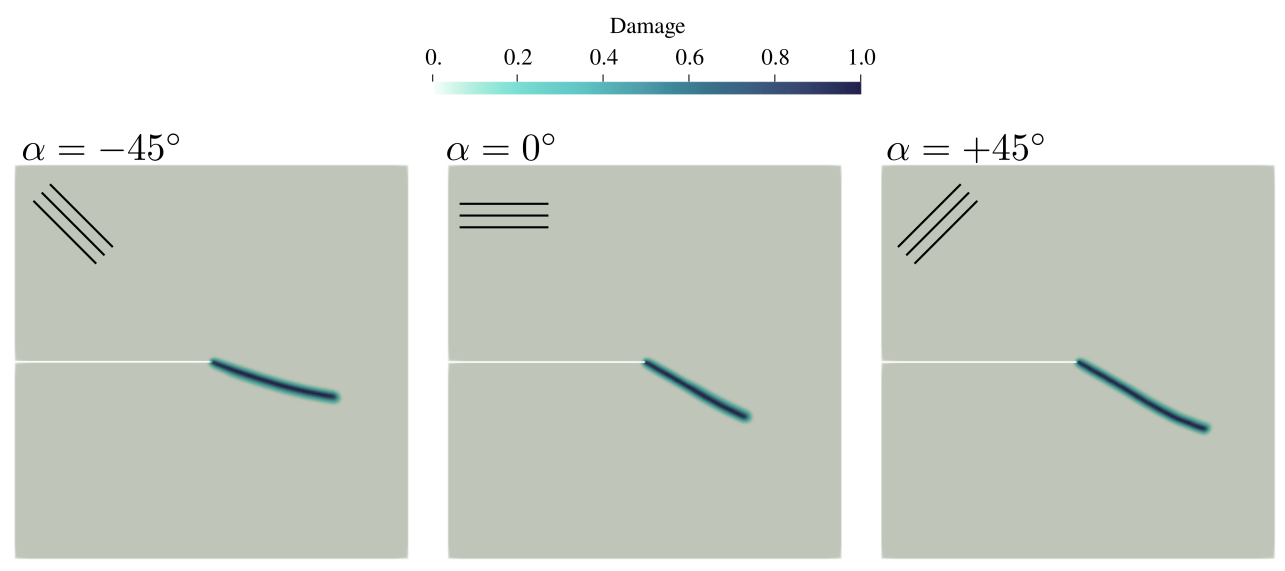

Figure 12: Crack path of the SD model for the mode II loading with varying principal orthotropic direction orientation

For $\alpha= \pm 45^{\circ}$, the LTD model predicts a crack at $-45^{\circ}$ for which the mode I component is much more important then the mode II component, contrary to what happens in the case of a horizontal crack propagation. However, depending on $\alpha$, the damage mode is either a transverse or a longitudinal crack. For the SD model, cracks propagate at a smaller angle and tend to curve during propagation contrary to the LTD model for which the crack orientation remains fixed during the propagation. Finally, in each cases, crack onset is characterized by a first unstable regime during which cracks jump abruptly to a finite length and are then followed by a stable propagation phase when increasing the applied displacement. This behavior is illustrated by the load-displacement curves obtained with the LTD model (Figure 13a) as well as by the evolution of the total crack length (Figure 13b). The SD model exhibits a similar behavior.

In both mode I and II examples, identical fracture energies were considered for both mechanisms. The picture obviously changes for different fracture energies, especially regarding transition between both mechanisms. This situation will now be examined in the next examples. 




(a) Load-displacement curves

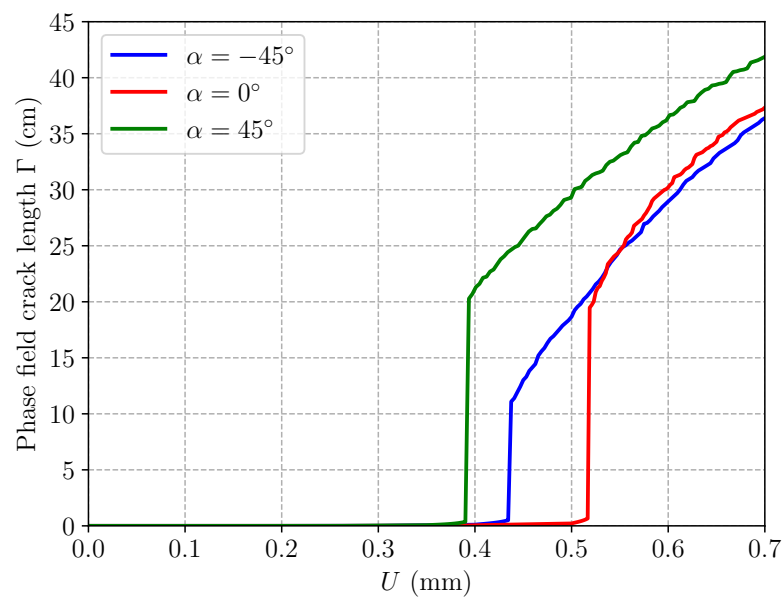

(b) Evolution of total phase-field crack length $\Gamma$

Figure 13: Evolution of macroscopic quantities for the mode II loading with varying principal orthotropic direction orientations (LTD model). Crack propagation is first characterized by an initial unstable phase corresponding to a finite-sized crack jump followed by a stable propagation phase.

\subsection{Crack kinking for $\alpha=90^{\circ}$}

By means of matched asymptotic expansions, Leguillon [62] determined the stress intensity factors (SIF) of a kinked crack in an anisotropic medium. For a crack loaded in pure mode I, the stress intensity factors $K_{\mathrm{I}}^{*}, K_{\mathrm{II}}^{*}$ in the kinked configuration for an infinitesimal kink length read as:

$$
K_{\mathrm{I}}^{*}=F_{11}(\varphi, \alpha) K_{\mathrm{I}}, \quad K_{\mathrm{II}}^{*}=F_{21}(\varphi, \alpha) K_{\mathrm{I}}
$$

where $K_{\mathrm{I}}$ is the mode I SIF before the kink and $F_{i j}$ are functions depending on the kink angle $\varphi$ and the orthotropy direction $\alpha$ as well as the relative elasticity moduli for a orthotropic medium. The corresponding energy release rate is then given by:

$$
G=A_{11}(\varphi, \alpha) K_{\mathrm{I}}^{2}
$$

where $A_{11}$ is obtained from the $F_{i j}$ functions.

Assuming an anisotropic fracture energy, a kinked crack will have to satisfy the following extension of Griffith's criterion, $G=G_{\mathrm{c}}(\varphi)$. Therefore, the kink angle $\varphi$ satisfies the following minimum principle:

$$
\frac{G_{\mathrm{c}}(\varphi)}{A_{11}(\varphi, \alpha)} \leq \frac{G_{\mathrm{c}}(\phi)}{A_{11}(\phi, \alpha)} \quad \forall \phi \in[-\pi ; \pi]
$$

Considering a situation of a mode I crack perpendicular to the fiber direction i.e. $\alpha=\pi / 2$ (see Figure 14) and a material for which all directions except $\alpha$ and $\alpha+\pi / 2$ are energetically 




Figure 14: Crack kinking problem with $\alpha=\pi / 2$. Depending on the ratio between vertical and horizontal fracture energies $G_{\mathrm{c}}^{2} / G_{\mathrm{c}}^{1}$, a mode I crack may either continue as a mode I crack or kink at $\pm 90^{\circ}$. The domain is $1 \mathrm{~m} \times 1 \mathrm{~m}$.

penalized in the anisotropic fracture energy $G_{\mathrm{c}}(\phi)$, a mode I crack will be able to kink at $\pm \pi / 2$ if the following criterion is met:

$$
\frac{G_{\mathrm{c}}(\pi / 2)}{A_{11}(\pi / 2, \pi / 2)} \leq \frac{G_{\mathrm{c}}(0)}{A_{11}(0, \pi / 2)}
$$

Material parameters of 62 are $E_{1}=142.1 \mathrm{GPa}, E_{2}=12.4 \mathrm{GPa}, \mu_{12}=2.425 \mathrm{GPa}$ and $\nu_{12}=0.531$ for which $A_{11}(\pi / 2, \pi / 2) / A_{11}(0, \pi / 2) \approx 0.09$ so that the previous kinking criterion reads in this case as $G_{\mathrm{c}}(\pi / 2) / G_{\mathrm{c}}(0)=G_{\mathrm{c}}^{2} / G_{\mathrm{c}}^{1} \leq 0.09$.

In order to assess the validity of the proposed phase-field approach for orthotropic materials, the same mode I problem as in subsection 5.1 has been considered using these new elastic constants and with varying values of the fracture energy ratio $\chi=G_{\mathrm{c}}^{2} / G_{\mathrm{c}}^{1}$. As predicted by (36), mode I cracks perpendicular to the fibers are observed for high values of $\chi$, whereas symmetric kinking of transverse cracks at $\pm 90^{\circ}$ is observed for sufficiently low values of $\chi$ (see Figure 15). More precisely, the transition between these two regimes is observed for $\chi$ between 0.1 and 0.105 . It is slightly larger than the critical value of $\chi_{\mathrm{c}}=0.09$ estimated by Leguillon. However, by reducing the value of the regularization length $\ell$, the transition level converges to the analytical value (see Table 3). This example clearly shows that the proposed LTD model is able to reproduce very well non trivial results of fracture mechanics in orthotropic materials.

The same problem has also been investigated with the SD model including an anisotropic fracture energy (26). Remarkably, irrespective of the value of $\chi$, this model leads always to a mode I crack propagation. No crack kinking has been observed, even for very small values 



Figure 15: Crack path of the LTD model $(\ell=10 \mathrm{~mm})$ for the kinking problem for varying values of $\chi=G_{\mathrm{c}}^{2} / G_{\mathrm{c}}^{1}$ : left $\chi=0.05$, middle $\chi=0.09$, right $\chi=0.11$.

\begin{tabular}{cc}
\hline Regularization length & Transition between kinking and mode I crack \\
\hline$\ell=10 \mathrm{~mm}$ & $0.1 \leq \chi_{\mathrm{c}} \leq 0.105$ \\
$\ell=7.5 \mathrm{~mm}$ & $0.095 \leq \chi_{\mathrm{c}} \leq 0.1$ \\
$\ell=5 \mathrm{~mm}$ & $0.09 \leq \chi_{\mathrm{c}} \leq 0.095$ \\
\hline
\end{tabular}

Table 3: Estimated transition threshold $\chi_{\mathrm{c}}$ between $90^{\circ}$ kinking $\left(\chi \leq \chi_{\mathrm{c}}\right)$ and mode I cracks $\left(\chi \geq \chi_{\mathrm{c}}\right)$ for varying regularization lengths with the LTD model. These results agree very well with the analytical estimate of $\chi_{\mathrm{c}} \approx 0.09$.

of $\chi$ such as 0.01. Such a model is therefore unable to simulate this non trivial behavior of cracks in orthotropic materials.

These results are further illustrated in Figure 16 which represents traction-displacement curves for both LTD and SD models with varying values of $\chi$. The LTD model predicts an unstable mode I crack propagation for $\chi \geq \chi_{\mathrm{c}}$ and a crack kinking of $90^{\circ}$ with a stable crack propagation for $\chi<\chi_{\mathrm{c}}$. On the contrary, regardless the value of $\chi$, the SD model always predicts an unstable mode I crack. During the kinked crack propagation, only a small amount of energy is dissipated, due to the low value of the $G_{\mathrm{c}}^{2}$ fracture energy and the smooth fracture evolution.

Finally, Leguillon also noticed that in the case of a positive $T$-stress, the kinked crack propagates in an unstable manner, whereas for a negative $T$-stress, the kink propagates in a stable manner. By adding an horizontal tensile or compressive load on the left boundary, we were able to verify such a feature with the LTD model. 




Figure 16: Traction-displacement curves for mode I loading with anisotropic fracture energy for varying fracture energy ratios $\chi=G_{\mathrm{c}}^{2} / G_{\mathrm{c}}^{1}(\ell=10 \mathrm{~mm})$. Solid lines correspond to the LTD model, dashed lines to the SD model including anisotropic fracture energy $(26)$.

\subsection{Bending of a notched beam}

This example considers the case of a beam of length $2 \mathrm{~m}$ and height $20 \mathrm{~cm}$, containing a prenotch of length $2.5 \mathrm{~cm}$ located on the bottom side at the middle of the beam. The principal orthotropic direction is aligned along the horizontal direction and the beam is loaded by an imposed displacement on its lateral sides combining extension and rotation: $\boldsymbol{u}(t)=\left(-u_{0}+\omega(t) y\right) \boldsymbol{e}_{x}$ on the left part and a symmetric displacement on the right part (see Figure 17). The imposed extension $u_{0}=0.1 \mathrm{~mm}$ is kept fixed during the simulation and the imposed rotation $\omega(t)$ is progressively increased until $0.002 \mathrm{rad}$. Elastic properties are those of Table 1 with $G_{\mathrm{c}}^{2}=50 \mathrm{~N} / \mathrm{m}, G_{\mathrm{c}}^{1}=10 G_{\mathrm{c}}^{2}=500 \mathrm{~N} / \mathrm{m}$ and $\ell=5 \mathrm{~mm}$.

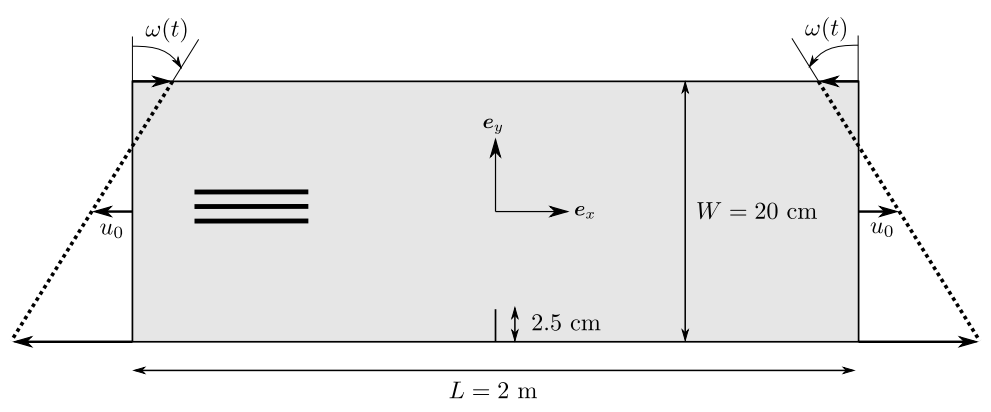

Figure 17: Bending of a notched beam: geometry and loading conditions

For this problem, damage evolution is first characterized by a kinked transverse crack propagating along the horizontal direction in accordance with the previous example (Figure 




(a) at $\omega=0.001$

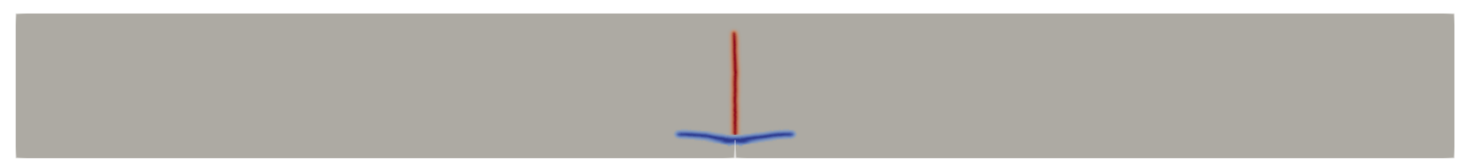

(b) at $\omega=0.002$

Figure 18: Damage field evolution during bending of a notched beam

18a). This first delamination phase is stable and stops at a certain load level. At this point, damage evolution is characterized by an unstable growth of a longitudinal crack along the vertical direction (Figure 18b). It is worth noting that during this unstable phase, the alternate minimization algorithm exhibits intermediate solutions in which both damage variables evolve simultaneously. However, the final equilibrium solution found by this process corresponds to an evolution of the longitudinal damage variable $d_{1}$ only.

\subsection{Open hole in tension}

In this last example, we investigate the problem of a plate of length $L=80 \mathrm{~mm}$ and width $W=18 \mathrm{~mm}$ containing a circular hole of radius $R=2.5 \mathrm{~mm}$. The plate is made of a unidirectional fiber-reinforced composite ply, with fibers making an angle $\alpha$ with respect to the vertical direction (Figure 19). In [63, 64], the plate effective strength was determined experimentally and compared to analytical or numerical predictions using coupled stressenergy criteria in the Finite Fracture Mechanics (FFM) framework.

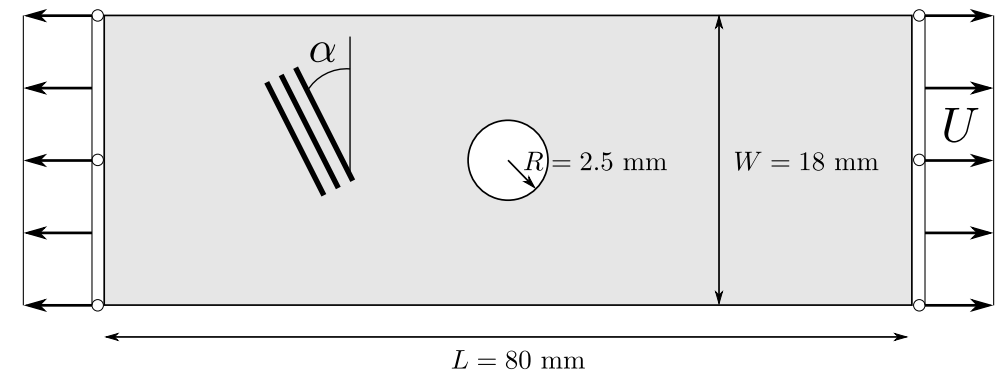

Figure 19: Plate with circular hole in tension

This problem has been simulated using our phase-field approach with both LTD and 
SD models. The regularization length for both models has been chosen as $\ell=1.5 \mathrm{~mm}$ corresponding to a uniaxial stress perpendicular to the fiber direction of $20.1 \mathrm{MPa}$ for both models, to be compared to the interlaminar strength of $20.25 \mathrm{MPa}$ measured in [63]. It has to be noted that, in this problem, the scale separation between the structural characteristic length (e.g. $W$ or $R$ ) and the regularization parameter $\ell$ is not verified since $\ell$ is of the order of $R$. As a consequence, the interpretation of the damage gradient model as an approximate representation of brittle fracture (12) does not hold in this case. However, it can still be considered as a damage model and used to predict the strength of the considered structure. The principal (fiber) toughness $G_{\mathrm{c}}^{1}$ has been chosen as 10 times greater than the secondary (matrix) toughness $G_{\mathrm{c}}^{2}$ since no data was available and since only transverse cracking has been observed experimentally.

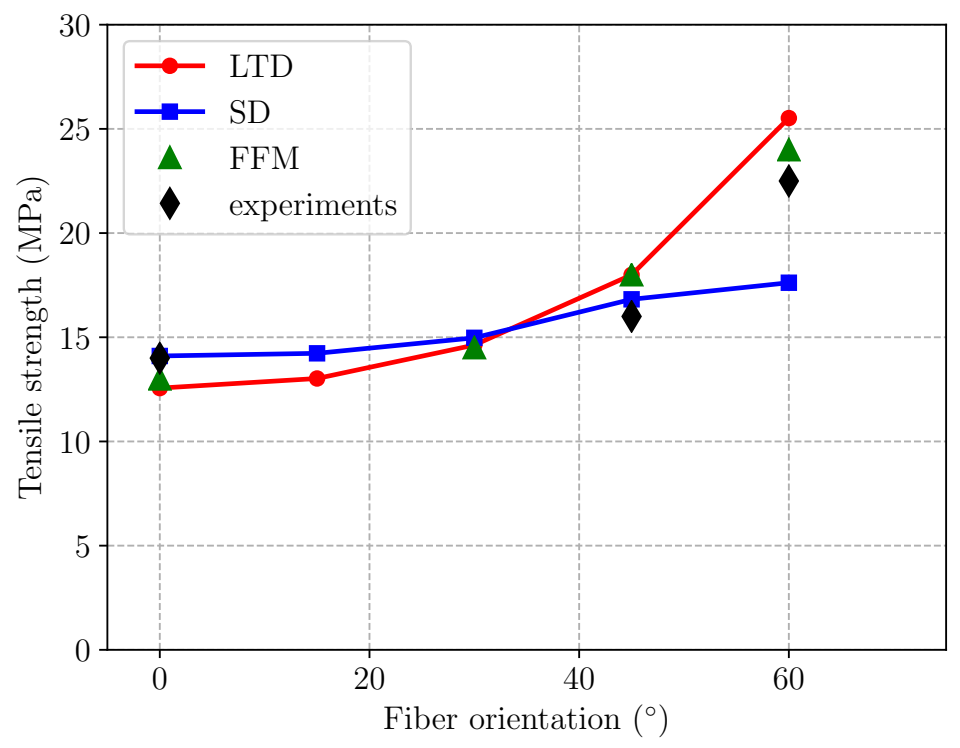

Figure 20: Comparison of effective strength predictions for the open hole problem: green triangles correspond to FFM computations [64] and black diamonds to experimental measurements of [63].

The predicted effective tensile strength by both phase-field approaches are represented in Figure 20 along with the FFM and experimental results. Although the SD model seems to be in good agreement for low values of fibers orientation, it is clearly unable to reproduce the increase of effective strength for an increasing fibers orientation. Conversely, the LTD model predictions are in relatively good agreement with the experimental measurements and, even more, with the FFM estimates. In particular, it reproduces the increase of effective strength with increasing values of $\alpha$, although it slightly overestimates the strength for the largest value. This can be attributed to the fact that the model does not consider a different mode II fracture energy from the mode I value. However, experiments showed that mode II fracture energy is in fact smaller $\left(G_{\mathrm{c}, \mathrm{II}}=472 \mathrm{~N} / \mathrm{m}\right)$ than the mode I value $\left(G_{\mathrm{c}, \mathrm{I}}=622 \mathrm{~N} / \mathrm{m}\right)$. A more refined model, for instance using a third damage variable associated with a shear 
failure mechanism, would be needed to represent this aspect which becomes important for large inclination angles. Besides, it must also be noted that the computed crack paths are not aligned with the fiber direction, contrary to experimental observations, but tend to be somewhere in between the fiber and the vertical direction. This may be attributed to the fact that the gradient term in the fracture energy is still isotropic and does not penalize any crack direction, contrary to the SD model with anisotropic fracture energy. Therefore, a more thorough analysis of the LTD model predictions remains to be done to decide whether the gradient term should also be anisotropic.

\section{Conclusions and perspectives}

This work is a first attempt at extending the phase-field approach to the simulation of anisotropic elastic brittle materials, such as, for instance, fiber-reinforced composites or wooden materials. More precisely, we focused on crack propagation of an orthotropic elastic medium in which two separate failure mechanisms are identified, namely longitudinal and transverse failure. Both mechanisms are represented by a single damage or phase-field variable and are associated with a corresponding fracture energy and, possibly, regularization length. The standard phase-field approach has therefore been extended to a setting including multiple damage mechanisms, each of them governed by its own, variationally derived, evolution law and damage criterion. In this first work, illustrative numerical applications have been dedicated only to a two-dimensional orthotropic material although the general framework, discussed in section 2, can be easily tailored to other more complex situations involving mechanisms with different fracture energy such as tension/compression damage, mode mixity in anisotropic rocks, etc.

Illustrative applications have demonstrated that the proposed phase-field model with two damage variables (LTD model) is a better candidate at representing the complex features of crack propagation in orthotropic media than would be a model including only one variable with an anisotropic fracture energy (SD model). More precisely, the LTD model is able to reproduce analytical fracture mechanics predictions of transition to $90^{\circ}$ kinking whereas the SD model is completely unable to do so. Finally, critical stresses for open-hole specimen in tension have been well reproduced using the LTD model compared to Finite Fracture Mechanics and experimental results.

Nevertheless, a more thorough comparison of the model predictions with other material models and experimental results is still needed. For instance, concerning unidirectional composite materials, this task is quite challenging because of the complex phenomena involved in the failure process, including plasticity in shearing conditions, fiber/matrix debonding and dependence of the fracture energy on mode mixity as previously mentioned. For all these reasons, the present work did not aim at simulating accurately all features of fracture in composites or anisotropic materials, but, instead, providing a general framework upon which more complex constitutive behaviors could be included in the future. For instance, interfacial energetic contributions have already been considered in previous works using the 
phase-field approach [21, 43, 65, 66] and could be used to model debonding between fibers and matrix at the unidirectional ply scale.

Secondly, on a more theoretical point of view, many questions still need to be answered such as the possibility of proving a $\Gamma$-convergence result towards a well-defined discontinuity model, the precise identification of crack orientation selection mechanisms 61 or stability of kinked configurations [67]. Finally, it has recently been demonstrated in [23] that variational phase-field models predict nucleation loads that smoothly vary from those predicted by a strength criterion to those predicted by a toughness criterion depending on the level of the stress singularity at notches, which is consistent with Leguillon's coupled criterion of Finite Fracture Mechanics. Although the open-hole example suggests that a similar result also holds for our model, a more detailed analysis on this aspect would certainly be valuable.

\section{Acknowledgement}

J. Bleyer would like to thank Prof. D. Leguillon for fruitful discussions related to this work. R. Alessi acknowledges the financial support of the MATHTECH-CNR-INdAM project.

\section{Appendix A. Derivation of the evolution laws}

From (4), the following first-order directional stability condition is easily derived from a vanishing $h$ :

$$
\left.\frac{\mathrm{d}}{\mathrm{d} h} \mathcal{E}(\boldsymbol{u}+h \delta \boldsymbol{u}, \underline{d}+h \delta \underline{d} ; t)\right|_{h=0} \geq 0 \quad \forall(\delta \boldsymbol{u}, \delta \underline{d}) \text { admissible }
$$

By making the expression of the total energy explicit through (1), we obtain:

$$
\begin{aligned}
& \int_{\Omega} \boldsymbol{\sigma}: \nabla^{s} \delta \boldsymbol{u} \mathrm{d} \Omega-\int_{\partial \Omega_{T}} \boldsymbol{F} \cdot \delta \boldsymbol{u} \mathrm{d} S+ \\
& \sum_{i=1}^{n} \int_{\Omega}\left(\partial_{d_{i}} \psi\left(\nabla^{s} \boldsymbol{u}, \underline{d}\right) \delta d_{i}+w_{i}^{\prime}\left(d_{i}\right) \delta d_{i}+w_{i}(1) \ell_{i}^{2} \nabla d_{i} \cdot \nabla \delta d_{i}\right) \mathrm{d} \Omega \geq 0
\end{aligned}
$$

with $\boldsymbol{\sigma}=\mathbb{C}(\underline{d}): \nabla^{s} \boldsymbol{u}$.

The equilibrium (6) and natural boundary conditions (7) are then obtained by standard arguments considering $\delta d_{i}=0$ for all $i$. Similarly, the damage evolution conditions (8)-(9) are obtained by considering $\delta \boldsymbol{u}=0, \delta d_{j}=0$ for $i \neq j$ and performing an integration by parts on the gradient term.

[1] G. A. Francfort, J.-J. Marigo, Revisiting brittle fracture as an energy minimization problem, Journal of the Mechanics and Physics of Solids 46 (1998) 1319-1342.

[2] A. Karma, D. A. Kessler, H. Levine, Phase-field model of mode III dynamic fracture, Physical Review Letters 87 (2001) 045501. 
[3] A. Karma, A. E. Lobkovsky, Unsteady crack motion and branching in a phase-field model of brittle fracture, Physical Review Letters 92 (2004) 245510.

[4] K. Pham, H. Amor, J.-J. Marigo, C. Maurini, Gradient damage models and their use to approximate brittle fracture, International Journal of Damage Mechanics 20 (2011) 618-652.

[5] P. Sicsic, J.-J. Marigo, From gradient damage laws to Griffith's theory of crack propagation, Journal of Elasticity 113 (2013) 55-74.

[6] C. Miehe, S. Mauthe, Phase field modeling of fracture in multi-physics problems. part III. crack driving forces in hydro-poro-elasticity and hydraulic fracturing of fluid-saturated porous media, Computer Methods in Applied Mechanics and Engineering 304 (2016) 619-655.

[7] C. Miehe, S. Mauthe, S. Teichtmeister, Minimization principles for the coupled problem of DarcyBiot-type fluid transport in porous media linked to phase field modeling of fracture, Journal of the Mechanics and Physics of Solids 82 (2015) 186-217.

[8] F. Freddi, G. Royer-Carfagni, Variational fracture mechanics to model compressive splitting of masonrylike materials, Annals of Solid and Structural Mechanics 2 (2011) 57-67.

[9] M. Hossain, C.-J. Hsueh, B. Bourdin, K. Bhattacharya, Effective toughness of heterogeneous media, Journal of the Mechanics and Physics of Solids 71 (2014) 15-32.

[10] T. Nguyen, J. Yvonnet, Q.-Z. Zhu, M. Bornert, C. Chateau, A phase field method to simulate crack nucleation and propagation in strongly heterogeneous materials from direct imaging of their microstructure, Engineering Fracture Mechanics 139 (2015) 18-39.

[11] Y. Xie, O. G. Kravchenko, R. B. Pipes, M. Koslowski, Phase field modeling of damage in glassy polymers, Journal of the Mechanics and Physics of Solids 93 (2016) 182-197.

[12] R. Alessi, M. Ambati, T. Gerasimov, S. Vidoli, L. De Lorenzis, Comparison of phase-field models of fracture coupled with plasticity, in: Advances in Computational Plasticity, Springer, 2018, pp. 1-21.

[13] F. Freddi, G. Royer-Carfagni, Phase-field slip-line theory of plasticity, Journal of the Mechanics and Physics of Solids 94 (2016) 257-272.

[14] R. Alessi, J.-J. Marigo, S. Vidoli, Gradient damage models coupled with plasticity: variational formulation and main properties, Mechanics of Materials 80 (2015) 351-367.

[15] M. Ambati, T. Gerasimov, L. De Lorenzis, Phase-field modeling of ductile fracture, Computational Mechanics 55 (2015) 1017-1040.

[16] M. Ambati, R. Kruse, L. De Lorenzis, A phase-field model for ductile fracture at finite strains and its experimental verification, Computational Mechanics 57 (2016) 149-167.

[17] M. J. Borden, T. J. Hughes, C. M. Landis, A. Anvari, I. J. Lee, A phase-field formulation for fracture in ductile materials: Finite deformation balance law derivation, plastic degradation, and stress triaxiality effects, Computer Methods in Applied Mechanics and Engineering 312 (2016) 130-166.

[18] C. Miehe, F. Aldakheel, A. Raina, Phase field modeling of ductile fracture at finite strains: A variational gradient-extended plasticity-damage theory, International Journal of Plasticity 84 (2016) 1-32.

[19] P. Sicsic, J.-J. Marigo, C. Maurini, Initiation of a periodic array of cracks in the thermal shock problem: a gradient damage modeling, Journal of the Mechanics and Physics of Solids 63 (2014) 256-284.

[20] A. Mesgarnejad, B. Bourdin, M. Khonsari, A variational approach to the fracture of brittle thin films subject to out-of-plane loading, Journal of the Mechanics and Physics of Solids 61 (2013) 2360-2379.

[21] A. L. Baldelli, J.-F. Babadjian, B. Bourdin, D. Henao, C. Maurini, A variational model for fracture and debonding of thin films under in-plane loadings, Journal of the Mechanics and Physics of Solids 70 (2014) 320-348.

[22] R. Alessi, S. Vidoli, L. D. Lorenzis, Variational approach to fatigue phenomena with a phase-field model: the one-dimensional case, Engineering Fracture Mechanics 190 (2018) 53-73.

[23] E. Tanné, T. Li, B. Bourdin, J.-J. Marigo, C. Maurini, Crack nucleation in variational phase-field models of brittle fracture, Journal of the Mechanics and Physics of Solids 110 (2018) 80-99.

[24] K. Pham, K. Ravi-Chandar, C. Landis, Experimental validation of a phase-field model for fracture, International Journal of Fracture 1 (2017) 83-101.

[25] E. Lorentz, A nonlocal damage model for plain concrete consistent with cohesive fracture, International Journal of Fracture 207 (2017) 123-159. 
[26] F. Freddi, F. Iurlano, Numerical insight of a variational smeared approach to cohesive fracture, Journal of the Mechanics and Physics of Solids 98 (2017) 156-171.

[27] M. J. Borden, C. V. Verhoosel, M. A. Scott, T. J. Hughes, C. M. Landis, A phase-field description of dynamic brittle fracture, Computer Methods in Applied Mechanics and Engineering 217 (2012) 77-95.

[28] M. Hofacker, C. Miehe, Continuum phase field modeling of dynamic fracture: variational principles and staggered FE implementation, International Journal of Fracture 178 (2012) 113-129.

[29] T. Li, J.-J. Marigo, D. Guilbaud, S. Potapov, Gradient damage modeling of brittle fracture in an explicit dynamics context, International Journal for Numerical Methods in Engineering 108 (2016) 1381-1405.

[30] J. Bleyer, C. Roux-Langlois, J.-F. Molinari, Dynamic crack propagation with a variational phase-field model: limiting speed, crack branching and velocity-toughening mechanisms, International Journal of Fracture 204 (2017) 79-100.

[31] H. Henry, M. Adda-Bedia, Fractographic aspects of crack branching instability using a phase-field model, Physical Review E 88 (2013) 060401.

[32] J. Bleyer, J.-F. Molinari, Microbranching instability in phase-field modelling of dynamic brittle fracture, Applied Physics Letters 110 (2017) 151903.

[33] V. Hakim, A. Karma, Crack path prediction in anisotropic brittle materials, Physical review letters 95 (2005) 235501.

[34] B. Li, C. Peco, D. Millán, I. Arias, M. Arroyo, Phase-field modeling and simulation of fracture in brittle materials with strongly anisotropic surface energy, International Journal for Numerical Methods in Engineering 102 (2015) 711-727.

[35] S. Teichtmeister, D. Kienle, F. Aldakheel, M.-A. Keip, Phase field modeling of fracture in anisotropic brittle solids, International Journal of Non-Linear Mechanics 97 (2017) 1-21.

[36] J. Clayton, J. Knap, Phase field modeling of directional fracture in anisotropic polycrystals, Computational Materials Science 98 (2015) 158-169.

[37] T.-T. Nguyen, J. Rethore, J. Yvonnet, M.-C. Baietto, Multi-phase-field modeling of anisotropic crack propagation for polycrystalline materials, Computational Mechanics 60 (2017) 289-314.

[38] X. Zhang, S. W. Sloan, C. Vignes, D. Sheng, A modification of the phase-field model for mixed mode crack propagation in rock-like materials, Computer Methods in Applied Mechanics and Engineering 322 (2017) $123-136$.

[39] P. Ladevèze, A damage computational method for composite structures, Computers \& Structures 44 (1992) 79-87.

[40] A. Matzenmiller, J. Lubliner, R. Taylor, A constitutive model for anisotropic damage in fibercomposites, Mechanics of materials 20 (1995) 125-152.

[41] P. Maimí, P. P. Camanho, J. Mayugo, C. Dávila, A continuum damage model for composite laminates: Part I-constitutive model, Mechanics of Materials 39 (2007) 897-908.

[42] B. Nedjar, N. Kotelnikova-Weiler, I. Stefanou, Modeling of unidirectional fibre-reinforced composites under fibre damage, Mechanics Research Communications 56 (2014) 115-122.

[43] R. Alessi, F. Freddi, Phase-field modelling of failure in hybrid laminates, Composite Structures 181 (2017) 9-25.

[44] J.-J. Marigo, C. Maurini, K. Pham, An overview of the modelling of fracture by gradient damage models, Meccanica 51 (2016) 3107-3128.

[45] B. Bourdin, G. A. Francfort, J.-J. Marigo, The variational approach to fracture, Journal of elasticity 91 (2008) 5-148.

[46] K. Pham, J.-J. Marigo, Approche variationnelle de l'endommagement: I. les concepts fondamentaux, Comptes Rendus Mécanique 338 (2010) 191-198.

[47] K. Pham, J.-J. Marigo, Approche variationnelle de l'endommagement: II. les modèles à gradient, Comptes Rendus Mécanique 338 (2010) 199-206.

[48] K. Pham, J.-J. Marigo, C. Maurini, The issues of the uniqueness and the stability of the homogeneous response in uniaxial tests with gradient damage models, Journal of the Mechanics and Physics of Solids 59 (2011) 1163-1190. 
[49] B. Bourdin, G. A. Francfort, J.-J. Marigo, Numerical experiments in revisited brittle fracture, Journal of the Mechanics and Physics of Solids 48 (2000) 797-826.

[50] A. Braides, Approximation of free-discontinuity problems, Springer-Verlag, 1998.

[51] E. Lorentz, S. Cuvilliez, K. Kazymyrenko, Convergence of a gradient damage model toward a cohesive zone model, Comptes Rendus Mécanique 339 (2011) 20-26.

[52] F. Freddi, G. Royer-Carfagni, Regularized variational theories of fracture: A unified approach, Journal of the Mechanics and Physics of Solids 58 (2010) 1154-1174.

[53] A. Logg, K.-A. Mardal, G. Wells, Automated solution of differential equations by the finite element method: The FEniCS book, volume 84, Springer Science \& Business Media, 2012.

[54] M. Alnæs, J. Blechta, J. Hake, A. Johansson, B. Kehlet, A. Logg, C. Richardson, J. Ring, M. E. Rognes, G. N. Wells, The FEniCS project version 1.5, Archive of Numerical Software 3 (2015) 9-23.

[55] T. Munson, J. Sarich, S. Wild, S. Benson, L. C. McInnes, Tao 2.0 users manual, 2012.

[56] S. Balay, S. Abhyankar, M. F. Adams, J. Brown, P. Brune, K. Buschelman, L. Dalcin, V. Eijkhout, W. D. Gropp, D. Kaushik, M. G. Knepley, L. C. McInnes, K. Rupp, B. F. Smith, S. Zampini, H. Zhang, H. Zhang, PETSc Web page, http://www.mcs.anl.gov/petsc, 2016.

[57] H. Petryk, Incremental energy minimization in dissipative solids, Comptes Rendus Mecanique 331 (2003) 469-474.

[58] G. Lancioni, Modeling the Response of Tensile Steel Bars by Means of Incremental Energy Minimization, Journal of Elasticity 121 (2015) 25-54.

[59] J. Bleyer, Phase-Field Composites : supplementary code for "Phase-field approach to anisotropic brittle fracture including several damage mechanisms", Zenodo, 2018. doi $10.5281 /$ zenodo.1188970.

[60] V. Hakim, A. Karma, Laws of crack motion and phase-field models of fracture, Journal of the Mechanics and Physics of Solids 57 (2009) 342-368.

[61] A. Chambolle, G. A. Francfort, J.-J. Marigo, When and how do cracks propagate?, Journal of the Mechanics and Physics of Solids 57 (2009) 1614-1622.

[62] D. Leguillon, Asymptotic and numerical analysis of a crack branching in non-isotropic materials, European journal of mechanics. A. Solids 12 (1993) 33-51.

[63] J. Modniks, E. Spārniņš, J. Andersons, W. Becker, Analysis of the effect of a stress raiser on the strength of a UD flax/epoxy composite in off-axis tension, Journal of Composite Materials 49 (2015) 1071-1080.

[64] J. Felger, N. Stein, W. Becker, Mixed-mode fracture in open-hole composite plates of finite-width: an asymptotic coupled stress and energy approach, International Journal of Solids and Structures 122 (2017) $14-24$.

[65] A. A. L. Baldelli, B. Bourdin, J.-J. Marigo, C. Maurini, Fracture and debonding of a thin film on a stiff substrate: analytical and numerical solutions of a one-dimensional variational model, Continuum Mechanics and Thermodynamics 25 (2013) 243-268.

[66] R. Alessi, J. Ciambella, A. Paolone, Damage evolution and debonding in hybrid laminates with a cohesive interfacial law, Meccanica 52 (2017) 1079-1091.

[67] A. Chambolle, G. A. Francfort, J.-J. Marigo, Revisiting energy release rates in brittle fracture, Journal of Nonlinear Science 20 (2010) 395-424. 